\title{
Bloom formation and Turing patterns in an infochemical mediated multi-trophic plankton model
}

\author{
Tahani A.S. Al-Karkhi \\ Department of Mathematical Sciences, University of Essex, Wivenhoe Park, \\ Colchester $\mathrm{CO}_{4} 3 \mathrm{SQ}$, United Kingdom \\ Rudy Kusdiantara \\ Department of Mathematical Sciences, University of Essex, Wivenhoe Park, \\ Colchester CO4 $3 S Q$, United Kingdom
}

Theoretical Physics Laboratory, Theoretical High Energy Physics and Instrumentation Research Group, Faculty of Mathematics and Natural Sciences, Institut Teknologi Bandung, Bandung, 40132, Indonesia

Hadi Susanto

Department of Mathematical Sciences, University of Essex, Wivenhoe Park, Colchester CO4 3SQ, United Kingdom

Edward A. Codling

Department of Mathematical Sciences, University of Essex, Wivenhoe Park, Colchester $\mathrm{CO}_{4} 3 \mathrm{SQ}$, United Kingdom

\begin{abstract}
A two-species predator-prey plankton model is studied, where the grazing pressure of microzooplankton on phytoplankton is controlled through external infochemical mediated predation. The system stability is analyzed in order to explain the conditions for phytoplankton bloom formation and to explore system bifurcations. The interplay between the level of infochemicalmediated external predation and the phytoplankton carrying capacity is considered over a range of parameter values and the resultant system dynamics illustrated. The model is extended to include a spatial diffusion term leading to a reaction-diffusion system that is investigated by determining the Turing space of the model. Thereafter, bifurcation analysis of specific timeindependent patterns is explored. Trough time integration, the system is also shown to exhibit the potential for temporally varying spatial patterns.
\end{abstract}

Keywords: plankton model, predator-prey dynamics, reaction-diffusion model, spatio-temporal pattern formation, Turing mechanism.

\section{Introduction}

Phytoplankton are key primary producers in oceanic foodwebs and hence play an important role in the global production of fisheries, as well as indirectly supporting populations of higher trophic level species such as seabirds and marine mammals [Jennings et al., 2009]. In pelagic foodwebs, grazing microzooplankton are known to form a trophic link between nutritionally poor phytoplankton and mesozooplankton species such as copepods [Sherr \& Sherr, 1988; Klein et al., 1999]. In turn, copepod predation on microzooplankton is thought to facilitate phytoplankton bloom formation by relieving microzooplankton grazing pressure [Hansen et al., 1993]. 
At larger scales the ocean can be highly turbulent with macro-scale currents and flows dominating. However, at the micro-scale, pelagic planktonic micro-organisms typically experience a highly viscous (low Reynolds number) environment where the physical properties of the medium allow chemical gradients to persist, and for chemical cues to be reliably transmitted. As a consequence, chemosensory systems have evolved in many aquatic species [Vos et al., 2006; Pohnert et al., 2007], and chemically-mediated interactions between trophic levels are known to affect population structure, community organization, and ecosystem function [Hay, 2009]. For example, the biogenic trace gas dimethylsulphide (DMS), which is derived from the algal secondary metabolite dimethylsulphoniopropionate (DMSP), is known to affect the behaviour of many marine organisms including foraging seabirds [Nevitt et al., 1995] and marine mammals [Kowalewsky et al., 2006]. More specifically, copepods such as Temora longicornis have been shown to respond to DMS when searching for prey [Steinke et al., 2006]. Production of DMS, and other potential infochemicals, is known to increase when phytoplankton such as Emiliania huxleyi are grazed by microzooplankton [Wolfe \& Gordon, 2000; Steinke et al., 2002]. The role that infochemicals such as DMS potentially play in plankton population dynamics and community structure is subsequently of great interest, not least because DMS is also known to aid in climate regulation through the formation of aerosols and cloud condensation nuclei in the atmosphere [Charlson et al., 1987].

A simple plankton foodwebs model was developed in [Lewis et al., 2012] to explore infochemical mediated tri-trophic interactions between phytoplankton, microzooplankton, and mesozooplankton (copepods). In [Lewis et al., 2012] it was assumed that increased grazing by microzooplankton on phytoplankton led to an increase in the background concentration of infochemicals such as DMS, which in turn led to increased mortality of microzooplankton through infochemical mediated predation by copepods. In the absence of infochemical interactions, the model of [Lewis et al., 2012] reduces to the well-known Rosenzweig-MacArthur model [Rosenzweig, 1963]. A standard result with the Rosenzweig-MacArthur model [Rosenzweig, 1963] is that an increase in prey carrying capacity acts to destabilize what would otherwise be a stable system (via a Hopf bifurcation), a result often termed the 'paradox of enrichment' [Rosenzweig, 1963]. However, the inclusion of infochemical interactions in the model of [Lewis et al., 2012] was shown to have (at low to medium levels) a stabilizing effect on the system dynamics. Subsequently, a further increase in the effect of the infochemical term led to multiple stable equilibria and finally a stable peak in phytoplankton density at higher levels, corresponding to a phytoplankton bloom [Lewis et al., 2012]. Further model extensions explored the role of infochemicals in a more complex foodwebs model that included competing phytoplankton species [Lewis et al., 2013]; the inclusion of tri-trophic infochemical interactions was shown to allow both competing species to survive indefinitely, in contrast to standard Lotka-Volterra competition models [Kot, 2001].

As primary producers that fix carbon through photosynthesis, phytoplankton are entirely dependent on light and hence production usually only occurs in depths of up to 200m [Jennings et al., 2009]. The vertical distribution of phytoplankton can be highly heterogeneous, but empirical evidence has shown that profiles of infochemicals such as DMS correspond closely to the chlorophyll maxima [Steinke et al., 2002]. Copepods are known to migrate vertically to follow the distribution of their prey, and hence they may potentially use vertical gradients of infochemicals to locate and remain within profitable foraging patches. The basic model in [Lewis et al., 2012] was non-spatial. However, in [Lewis et al., 2013] it was shown that small increases in the ability of copepods to sense infochemicals can promote their persistence in the system, and hence increased sensitivity to infochemicals can act as an evolutionarily stable strategy. The scenario of vertically migrating zooplankton has been studied by including a spatial heterogeneity leading to reaction-advection-diffusion models, see, e.g., [Huisman \&et al., 2006; Zagaris et al., 2009; Zagaris \& Doelman, 2011; Sewalt et al., 2015].

Pattern formation in plankton systems can also be considered in the horizontal plane using classical reaction-diffusion approaches, including analysis of Turing patterns [Malchow et al., 2008; Feudel, 2008]. For example, [Ghorai \& Poria, 2016] showed how apparently chaotic patterns can emerge due to preypredator interactions in a system supplying additional food, while [Roelke et al., 1999] showed how a Turing instability can cause inhomogeneous distributions of nutrients leading to spatial patterns. Modelling approaches have also shown how spatial patchiness and heterogeneity can subsequently affect plankton dynamics and potential bloom formation [Hammer \& Pitchford, 2005; Pitchford \& Brindley, 1998]. Here 
we develop and explore the basic infochemical mediated predator-prey model of [Lewis et al., 2012] in two main ways. Firstly, we explore in more detail the role of carrying capacity and infochemical interaction on the model dynamics and potential for bloom formation. In their original study, [Lewis et al., 2012] only considered a single free exploratory parameter (the infochemical interaction term) and used a fixed carrying capacity. In addition, their study was mainly undertaken using numerical simulations for specific cases and a detailed stability and bifurcation analysis was not fully completed. Secondly, we extend the model of [Lewis et al., 2012] to include spatial diffusion terms corresponding to the horizontal plane. We undertake a Turing analysis of the reaction-diffusion system and explore the subsequent spatial pattern formation. In particular, we highlight how temporally-varying spatial patterns are possible within the system.

\section{Mathematical model}

\subsection{General model and description}

The model we consider is a modified version of that introduced by [Lewis et al., 2012] that includes a horizontal diffusion term:

$$
\begin{aligned}
\frac{\partial P}{\partial t} & =F_{\Delta}(P, M):=D_{P} \frac{\partial^{2} P}{\partial x^{2}}+r P\left(1-\frac{P}{K}\right)-\frac{a P M}{1+b P} \\
\frac{\partial M}{\partial t} & =G_{\Delta}(P, M):=D_{M} \frac{\partial^{2} M}{\partial x^{2}}+\frac{\gamma a P M}{1+b P}-m M-\nu \frac{a P M^{2}}{1+b P},
\end{aligned}
$$

where $P$ and $M$ are the densities of phytoplankton and microzooplankton in the closed homogeneous system. Following [Lewis et al., 2012], phytoplankton are assumed to grow logistically with intrinsic rate of growth $r$ and carrying capacity $K$, where the carrying capacity corresponds to nutrient limitation and self shading. Microzooplankton graze on phytoplankton according to a Holling type II functional response, where the rate of grazing saturates at high densities of phytoplankton; phytoplankton biomass is converted to microzooplankton biomass with efficiency $\gamma$. The parameter $m$ corresponds to microzooplankton mortality that occurs in the absence of infochemicals; microzooplankton mortality is assumed to be mainly caused by copepods but $m$ also accounts for mortality due to processes such as sinking and additional predation by other zooplankton or by higher trophic levels. The parameter $\nu$ (exactly equivalent to the $\lambda$ parameter in [Lewis et al., 2012]) corresponds to an increase in the predation of microzooplankton by copepods due to the instantaneous release of infochemicals when phytoplankton are grazed. Hence the term can represent both an increased copepod sensitivity and response and/or improved copepod search efficiency at higher chemical concentrations. Note that copepods and infochemicals are not included in the model directly as variables, but are both represented in the system through the interaction term involving $\nu$. Parameters $a$ and $b$ represent the clearance rate of microzooplankton at low food densities and its half-saturation density, respectively. Typical values of the parameters are summarised in Table 2.1. The main difference of our model from that of [Lewis et al., 2012] is the Laplacian terms with strength $D_{P}$ and $D_{M}$ that represent the diffusion of phytoplankton and microzooplankton, respectively.

\subsection{Model realism, assumptions and limitations}

The model of [Lewis et al., 2012] was deliberately simple to allow for analytical progress, while still capturing the key features of the biological system being considered. The key simplifying assumptions made by [Lewis et al., 2012] also apply to (1), in addition to several further assumptions that we introduce to deal with the spatial component of the model. Specifically, as mentioned above and following [Lewis et al., 2012], we assume an instantaneous release of infochemical when phytoplankton are grazed by microzooplankton. Subsequently an instantaneous response by predatory copepods to the localised absolute increase in infochemical concentration is also assumed. This is potentially an over-simplification of the biological reality since copepods are also known to follow differential gradients of infochemicals to find prey, potentially leading to higher encounter rates [Heuschele \& Selander, 2014]. As with [Lewis et al., 2012], we also assume that the copepod population is constant (in relative terms compared to the dynamics of phytoplankton and microzooplankton) which implies either a constant equilibrium level population is maintained, or that 
Table 1. Parameter values used in the model (1).

\begin{tabular}{|l|l|l|l|}
\hline \hline Parameter & Value & Unit & Source \\
\hline$r$ & $0-5$ & day $^{-1}$ & [Edwards, 1999] \\
$K$ & $0-1000$ & $\begin{array}{l}\mu g \\
I^{-1} \\
\mu g \\
C\end{array}$ & [Franks, 2002; Morozov, 2010] \\
& 0.3 & $\begin{array}{l}C I^{-1} \\
\text { day }^{-1} \\
\mu g\end{array}$ & [Saiz, 2007; Hansen, 1990] \\
$b$ & 0.05 & $\begin{array}{l}I^{-1} \\
\text { day }^{-1} \\
\text { day }^{-1} \\
\text { day }^{-1}\end{array}$ & [Saiz, 2007; Hansen, 1990] \\
$\gamma$ & 0.5 & 0.3 \\
$m$ & $0-0.2$ & \\
$\nu$ & & & \\
\hline
\end{tabular}

the model time-scales are of the order of a few days. The final assumption of [Lewis et al., 2012] that also applies to (1) is that microzooplankton provide the only trophic link between copepods and phytoplankton (i.e. copepods do not themselves feed directly on phytoplankton, although this can occur in scenarios where densities of microzooplankton are low [Calbet, 2001]). Since we extend the model of [Lewis et al., 2012] to include horizontal diffusion, we need to make further assumptions about the spatial domain and the relative movement of phytoplankton and microzooplankton. Firstly, we assume that turbulence effects are small and that currents or circulation features in the water column are absent so that plankton movement is governed by viscosity and the relative differences in swimming behaviour between phytoplankton and microzooplankton can be explored without being dominated by larger-scale oceanographic features. For the sake of analytical and numerical simplicity, we assume periodic boundary conditions (effectively infinite domain size), but we restrict the corresponding length of the computational domain to the order of $10 \mathrm{~s}$ of meters, given as a multiple of the wavelength of the eigenfunction corresponding to the most unstable eigenvalue (see Section 4). We assume that movement of phytoplankton and microzooplankton is governed by viscosity and is mainly driven by either passive diffusion due to low-level turbulence phytoplankton or through randomly oriented swimming microzooplankton; we do not include an advection term in the model, although microzooplankton may be able to orient directly towards prey using a range of search mechanisms and cues [Kiørboe et al., 2014]. We assume that $D_{P}<D_{M}$, which is biologically justified since most species of phytoplankton are non-motile and diffuse purely through passive drift due to turbulence and currents; in contrast, many microzooplankton species are highly motile and can swim horizontally or vertically through the water column, leading to a higher diffusive capability [Harvey \& Menden-Deuer, 2012; Kiørboe et al., 2014].

\subsection{Model parameterisation}

In the subsequent analysis, and following [Lewis et al., 2012], we consider the infochemical interaction mortality $\nu$ as our main exploratory parameter. However, we go further than [Lewis et al., 2012] and also consider the phytoplankton carrying capacity as an additional exploratory parameter. We otherwise parameterise the model according to the parameter values and ranges used in [Lewis et al., 2012], see Table 2.1. For simplicity, we fix $D_{M}=1$, corresponding to a relative diffusivity of approximately $1 \mathrm{~m}^{2} /$ day due to drift and randomly oriented swimming. We consider a range of values for $D_{P}$, where $D_{P} \ll D_{M}$ (since $P$ are typically non-motile), and explore how the subsequent spatial dynamics change given the difference in relative diffusivity between $M$ and $P$, see Section 4 .

\section{Analysis of the non-spatial model}

We first consider the mathematical model (1) in the absence of diffusion, i.e. $D_{P}=D_{M}=0$, which is exactly the case studied in [Lewis et al., 2012] who used continuation software to explore results numerically. In the following, we will present more detailed analytical results that explain the original findings of [Lewis et 


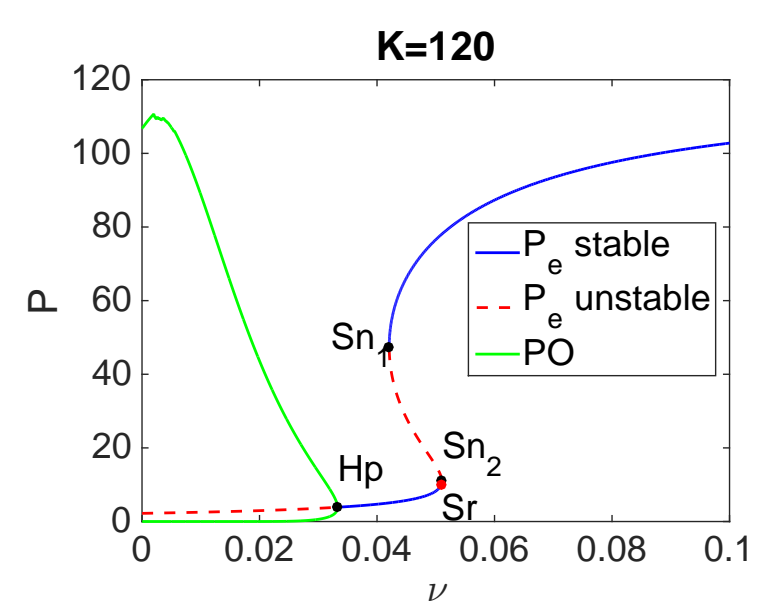

(a)

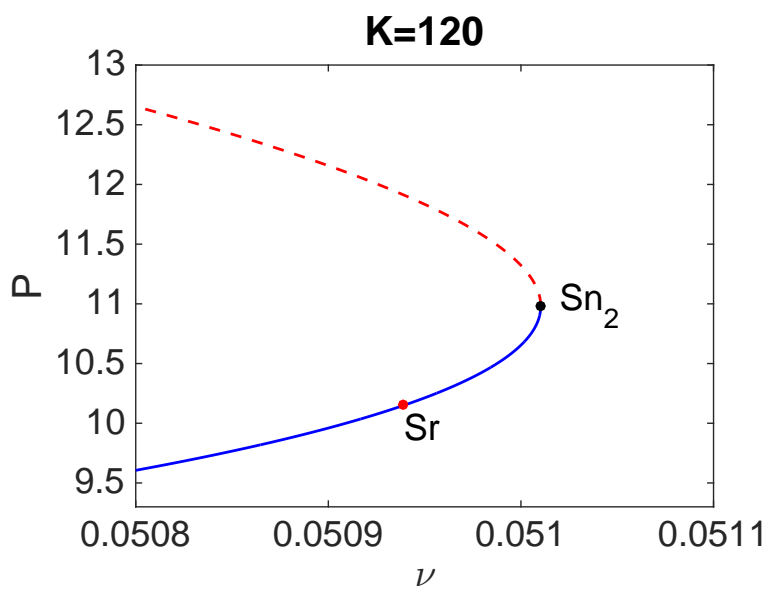

(c)

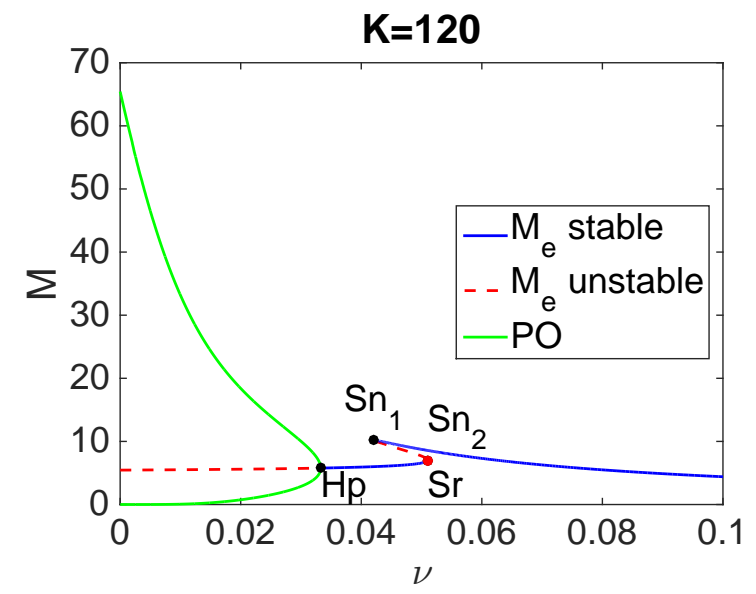

(b)

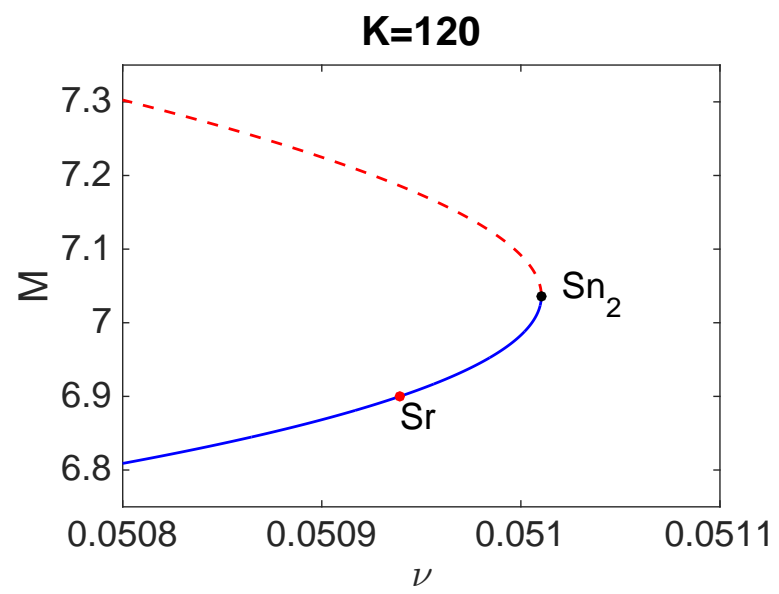

(d)

Fig. 1. Bifurcation diagram showing the co-existence equilibria, $P_{e}$ and $M_{e}$, and their associated stability and periodic orbits for $K=120$ and $r=1.5$. The other parameter values are given in Table 2.1. Panels 1(c)-1(d) are zoomed-in around specific points of interest corresponding to bifurcations. $H p$ indicates the Hopf bifurcation point, while $S n_{1}$ and $S n_{2}$ are the first and second saddle-node bifurcation. $S r$ shows the point when the eigenvalues of the uniform solution change from being complex into real valued. The green solid line indicates the maximum and minimum of the periodic orbit of the system.

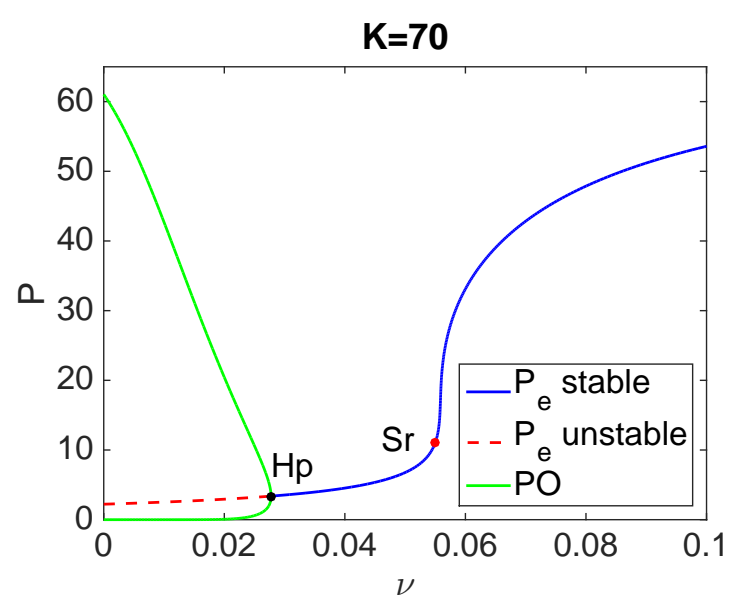

(a)

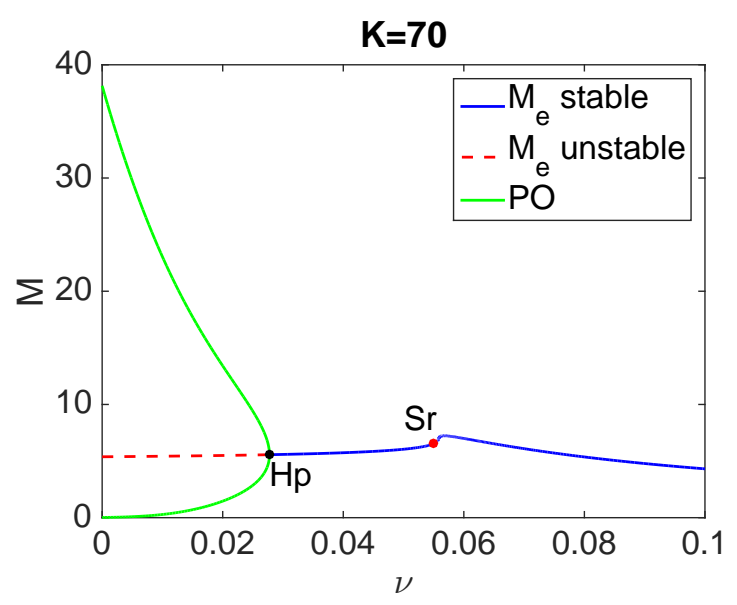

(b)

Fig. 2. The same picture as in Figs. 1(a) and 1(b), but for $K=70$. 
al., 2012]. The analysis in this section will also be used later when extending the model to a spatial context by including non-zero diffusion terms. In Sections 3.1 and 3.2 we determine analytical expressions for the location and stability of the equilibria in the general case, and illustrate results for the specific cases of $K=120$ and $K=70$, before extending this to cover all values of $0 \leq K \leq 1000$ in Section 3.3, see Table 2.1 for a complete list of parameter values and intervals.

\subsection{Location of equilibria}

The equilibrium points $P(t)=P_{e}$ and $M(t)=M_{e}$ of (1) corresponding to $d P / d t=d M / d t=0$ can be shown to include the trivial state $\left(P_{e, 0}, M_{e, 0}\right)=(0,0)$, the microzooplankton-free equilibrium $\left(P_{e, m f}, M_{e, m f}\right)=$ $(K, 0)$ and the co-existence state that satisfies the polynomial

$$
r b \nu P_{e}^{3}+r \nu(1-K b) P_{e}^{2}+K(\gamma a-m b-\nu r) P_{e}-m K=0
$$

and

$$
M_{e}=\frac{P_{e}(a \gamma-b m)-m}{\nu a P_{e}} .
$$

In general, the function (2) will have three roots. Following [Nickalls, 1993], they are given by

$$
P_{e, j+1}=\frac{K b-1}{3 b}+\frac{2}{3 b} \sqrt{(K b-1)^{2}-\frac{3 b K}{r \nu}(\gamma a-m b-\nu r)} \cos \left(\frac{\theta+2 j \pi}{3}\right), j=0,1,2,
$$

where $\theta=\cos ^{-1}\left(\frac{y_{N}}{h}\right)$,

$$
y_{N}=-r \nu \frac{(K b-1)^{3}}{9 b^{2}}+\frac{r \nu(K b-1)^{2}}{27 b^{2}}+\frac{K(\gamma a-m b-\nu r)(K b-1)}{3 b}-m K,
$$

and

$$
h=\frac{2 \nu r b}{27}\left(\frac{r \nu(1-K b)^{2}-3 b K(\gamma a-m b-\nu r)}{\nu r b^{2}}\right)^{\frac{3}{2}} .
$$

Two roots can merge into one when $y_{N}^{2}=h^{2}$ and then become complex valued afterwards.

The cubic shape of the solution given by (4) can be seen clearly in Fig. 1 for $K=120$ with $r=1.5$ and other parameter values as given in Table 2.1; note that Fig. 1(a) is exactly equivalent to Fig. 3 of [Lewis et al., 2012]. The number of coexistence equilibria in the system can be seen to clearly depend on the parameter values of $K$ and $\nu$ : for instance when $K=70$, as presented in Fig. 2, only a single real root of (4) exists for all values of $\nu$. The saddle-saddle bifurcation point, i.e., when the two saddle node bifurcation points collide, occurs at $K \approx 71.97349 \ldots$.

\subsection{Stability of equilibria}

To determine the linear stability of each equilibrium point we write

$$
\left(\begin{array}{c}
P(t) \\
M(t)
\end{array}\right)=\left(\begin{array}{c}
P_{e} \\
M_{e}
\end{array}\right)+\left(\begin{array}{c}
\epsilon_{1} \\
\epsilon_{2}
\end{array}\right) e^{\lambda t}
$$

From (5), a uniform solution is said to be linearly stable when $\lambda \leq 0$ and unstable otherwise. Substituting (5) into (1) and linearising about $\epsilon_{1}=\epsilon_{2}=0$, we obtain the linear eigenvalue problem

$$
\lambda\left(\begin{array}{c}
\epsilon_{1} \\
\epsilon_{2}
\end{array}\right)=\left(\begin{array}{ll}
a_{11} & a_{12} \\
a_{21} & a_{22}
\end{array}\right)\left(\begin{array}{l}
\epsilon_{1} \\
\epsilon_{2}
\end{array}\right)
$$

where

$$
\begin{aligned}
& a_{11}=r-\frac{2 r P_{e}}{K}-\frac{a M_{e}}{\left(1+b P_{e}\right)}+\frac{a b P_{e} M_{e}}{\left(1+b P_{e}\right)^{2}}, a_{12}=-\frac{a P_{e}}{1+b P_{e}}, \\
& a_{21}=-\frac{a M_{e}\left(\nu M_{e}-\gamma\right)}{\left(1+b P_{e}\right)^{2}}, a_{22}=-m-\frac{a P_{e}\left(2 M_{e} \nu-\gamma\right)}{1+b P_{e}} .
\end{aligned}
$$




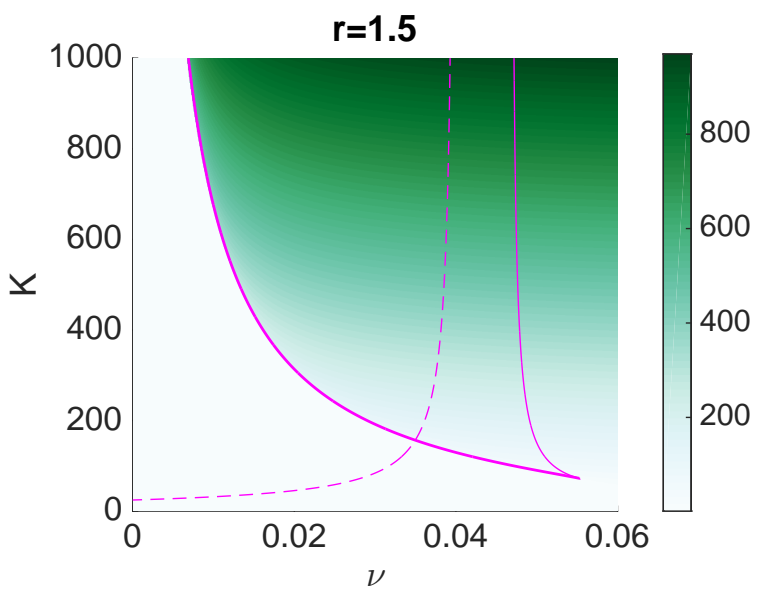

(a)

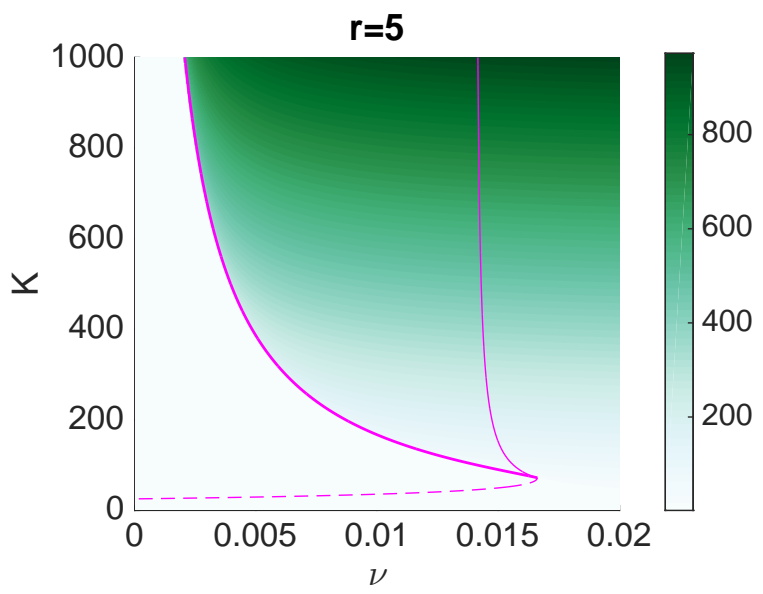

(c)

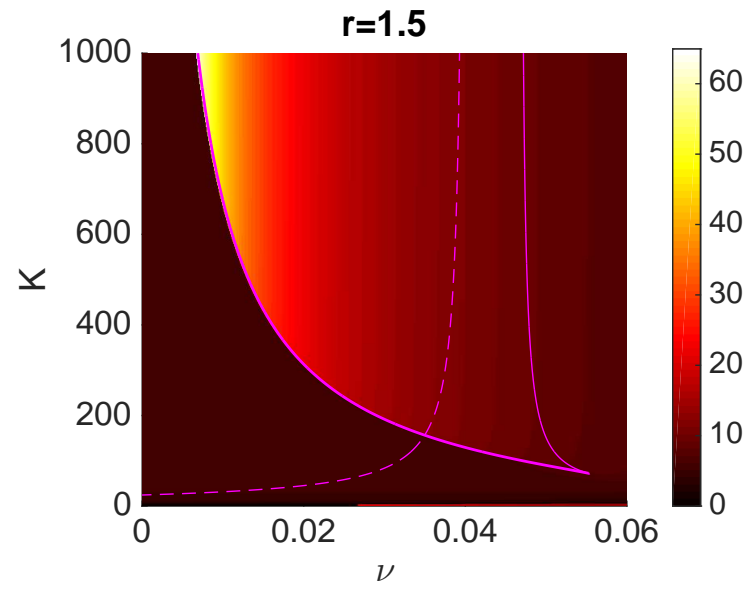

(b)

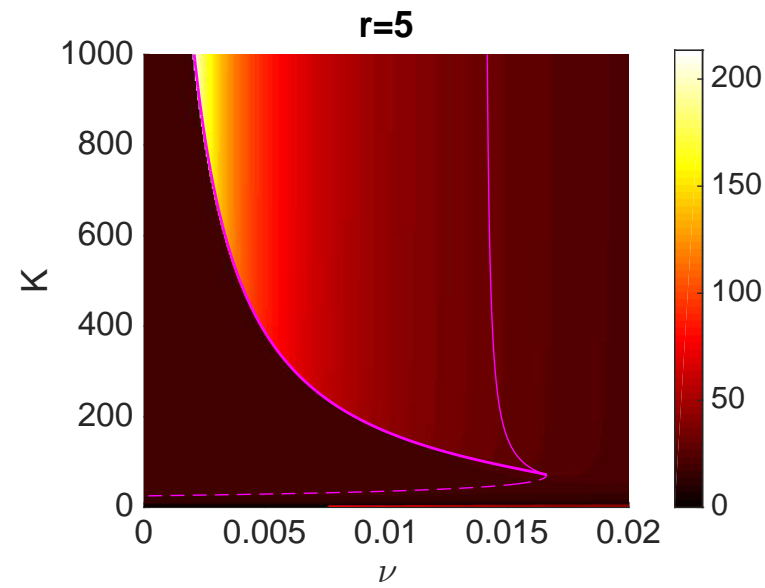

(d)

Fig. 3. Population densities of (a,c) $P_{e}$ and (b,d) $M_{e}$ corresponding to the largest coexistence state as $K$ and $\nu$ vary for $r=1.5$ and $r=5$. The other parameter values are the same as those in Fig. 1 . Bifurcation curves of the system as $K$ and $\nu$ vary are also plotted. The magenta dashed line corresponds to the Hopf bifurcation, $H p$, the magenta thick solid line to the first saddle-node bifurcation, $S n_{1}$, and the magenta thin solid line to the second saddle-node bifurcation, $S n_{2}$. Note that $S r$ is not marked as it is always very close to $S n_{2}$.

One can then readily obtain the eigenvalues

$$
\lambda_{ \pm}=\frac{1}{2}\left[a_{11}+a_{22} \pm \sqrt{\left(a_{11}-a_{22}\right)^{2}+4 a_{12} a_{21}}\right] .
$$

It is straightforward to show that the trivial state and the microzooplankton-free equilibrium are unstable (source) with the corresponding eigenvalues $\lambda_{ \pm}=r, m$ and $\lambda_{ \pm}=r, m+(\gamma a K) /(K b+1)$, respectively. The eigenvalues for the coexistence state are also found from (7); full analytic expressions are omitted due to length but the corresponding system stability is shown in Figs. 1 and 2, where stable solutions are denoted as solid lines (unstable as dashed lines).

As originally reported in [Lewis et al., 2012], for $K=120, r=1.5$, and other parameter values as given in Table 2.1, the system exhibits hysteresis. As the infochemical interaction parameter, $\nu$, initially increases from 0, a supercritical Hopf bifurcation $(H p)$ occurs, and the system moves from a stable limit cycle (shown by the green lines in Figs. 1(a)) around the unstable coexistence state to a single stable coexistence state. A subsequent saddle-node bifurcation $\left(S n_{1}\right)$ leads to a region with bistability (two stable and one unstable (saddle) coexistence states). The local stability of the stable equilibria changes (from a focus to a node) at the point $S r$, where the eigenvalues change from complex to real. At this point, we say 
that the system gains the monotonicity property, i.e., when the solution approaches a stable equilibrium in a monotonous manner (referred to as overdamped oscillations) [Jankovic \& Petrovskii, 2014]. Finally, a second saddle-node bifurcation $\left(S n_{2}\right)$ occurs, leaving only the larger stable coexistence state in the system. This was interpreted by [Lewis et al., 2012] as the point at which persistent phytoplankton bloom formation was possible (persistent bloom formation in this context meaning that $P_{e}$ is stable and approaches $K$ ).

When $K=70$ the solution to (2) and (3) allows for only a single real solution (and hence a single coexistence state) for all $\nu$. Although the initial dynamics for small $\nu$ are similar to $K=120$ (Hopf bifurcation), at larger values of $\nu$ the system no longer exhibits hysteresis or a region of bistability, as illustrated in Fig. 2. Nevertheless, as $\nu$ increases a rapid increase in $P_{e}$ occurs after the saddle-node bifurcation occurs and (persistent) bloom formation is possible.

\subsection{Varying the carrying capacity $K$}

In this section we go beyond [Lewis et al., 2012] (who only considered the case of $K=120$ ) and explore in more detail how the carrying capacity of the phytoplankton population, $0 \leq K \leq 1000$, affects the system dynamics and potential for bloom formation. Note that this range of $K$ may not be fully realistic depending on the biological context, but is considered to illustrate the range of system dynamics.

Figure 3 illustrates the densities of $P_{e}$ and $M_{e}$ corresponding to the largest coexistence state as $K$ and $\nu$ vary. The saddle-node bifurcation that corresponds to the point at which a phytoplankton bloom can form is clearly seen for large $K$ on both plots and is also highlighted with the red line in the figure (also compare to Fig. 1(a)). It is clear from Fig. 3(a) that for low $\nu$ values, persistent phytoplankton bloom formation is not possible (in such cases the system remains unstable and dynamics are analogous to the Rosenzweig-MacArthur model [Rosenzweig, 1963]). For values of $K$ and $\nu$ that lie above the bifurcation curve, it is clear that larger values of $K$ and $\nu$ simply lead to a higher density for $P_{e}$ as might be expected.

Figure 3 shows that for large $K, M_{e}$ is found to initially increase significantly as $\nu$ increases past the saddle-node bifurcation, before decreasing once $\nu$ increases further. This result is somewhat counterintuitive given that an increase in $\nu$ corresponds to higher mortality of microzooplankton due to increased copepod predation. This result can be related to the well-known 'hydra-effect' in predator-prey models [Sieber \& Hilker, 2012], but in this case has a simple explanation: an initial relief of grazing pressure allows $P$ to bloom, and in turn a higher population density of $P$ is able to support a higher population density of $M$.

Figure 3 also illustrates the general system stability as $K$ and $\nu$ vary for $r=1.5$ and $r=5$. Qualitatively similar dynamics are obtained for all values of the growth rate $r$, although for $r=5$ the Hopf bifurcation no longer crosses into the region of bistability between the two saddle-node bifurcations.

\section{Pattern formation in the model with diffusion}

In this section we consider the effect of diffusion in the horizontal direction on the system stability. Note that the presence of diffusion does not change the location and stability of the equilibrium as discussed for the non-spatial model in the previous sections, but may change how system perturbations develop depending on the relative diffusivity of $P$ and $M$.

\subsection{Analysis of Turing instability}

The stability of the equilibrium is obtained from taking the ansatz, instead of (6),

$$
\left(\begin{array}{c}
P(x, t) \\
M(x, t)
\end{array}\right)=\left(\begin{array}{c}
P_{e} \\
M_{e}
\end{array}\right)+\left(\begin{array}{c}
\epsilon_{1} \\
\epsilon_{2}
\end{array}\right) e^{\lambda t+i k x},
$$

from which we obtain, instead of (8), the dispersion relation

$$
\lambda(k)=\frac{1}{2}\left[a_{11}+a_{22}-\left(1+D_{P}\right) k^{2} \pm \sqrt{\left(a_{11}-a_{22}+k^{2}\left(1-D_{P}\right)\right)^{2}+4 a_{12} a_{21}}\right] .
$$

Here, $a_{i j}, i, j=1,2$ are still given by (7). Note that the stability of the equilibrium in the absence of diffusion (8) can be recovered from (10) by setting $k=0$. 


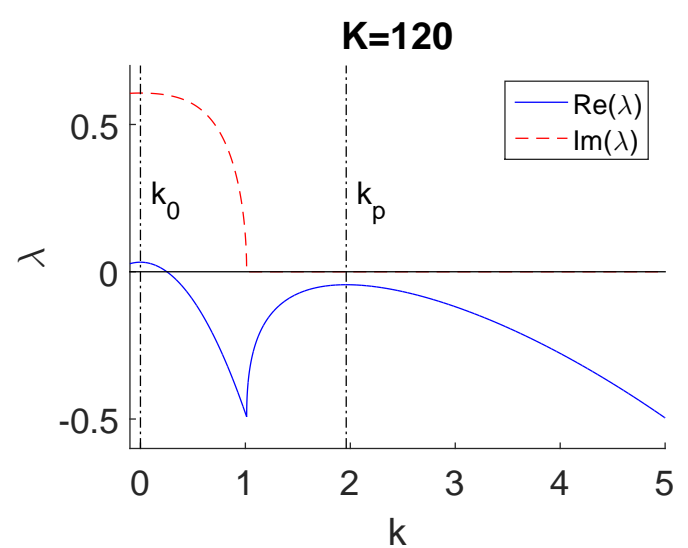

(a)

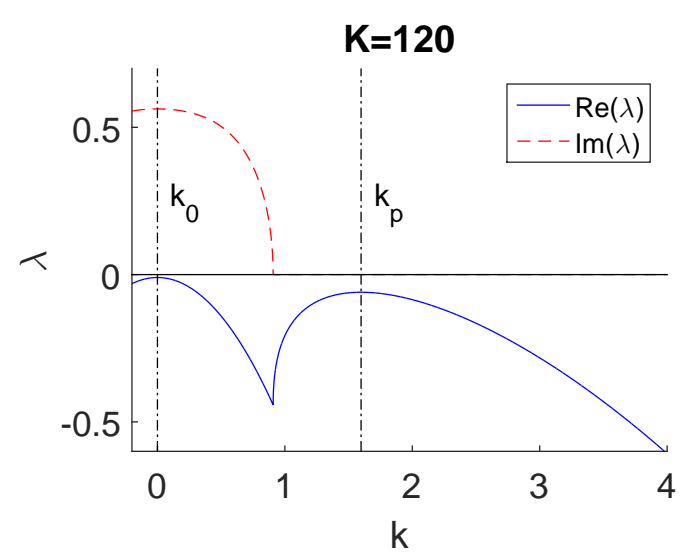

(c)

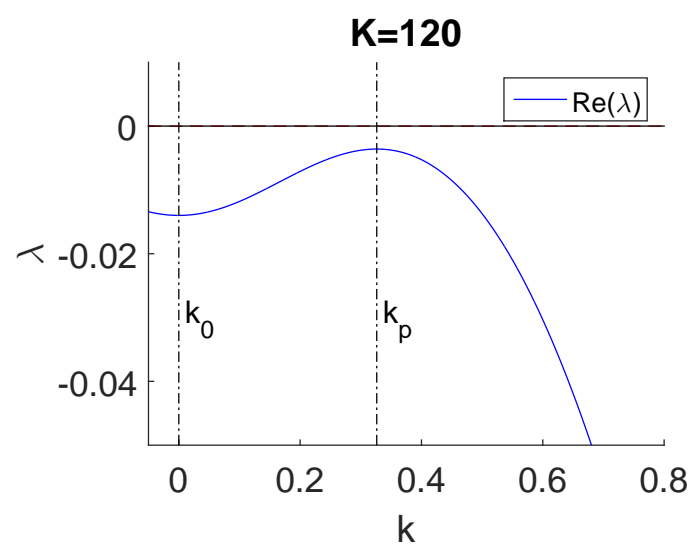

(e)

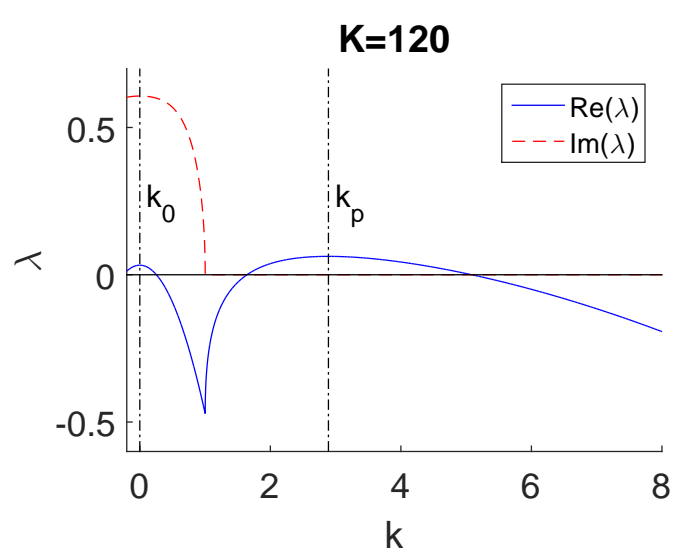

(b)

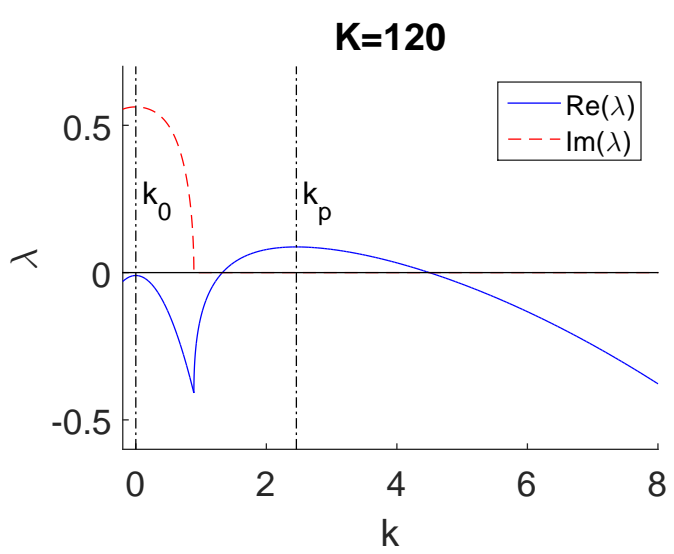

(d)

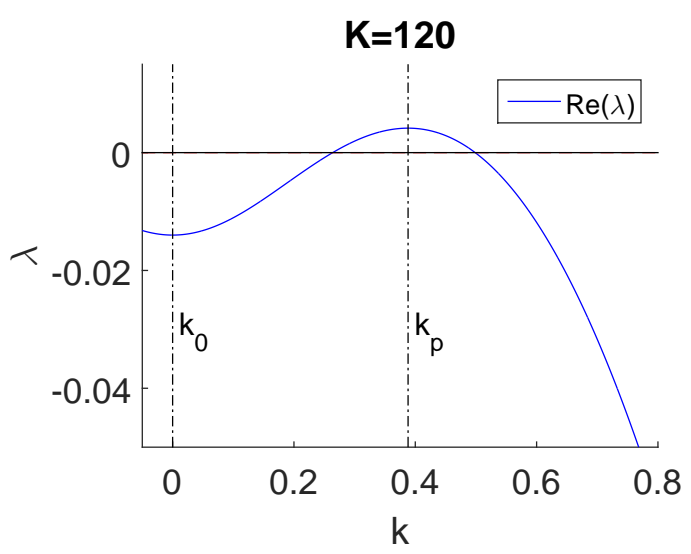

(f)

Fig. 4. The dispersion relation (10) of the coexistence equilibrium $P_{e, 1}, M_{e, 1}$ (see (4)) as a function of the perturbation wavenumber $k$ for parameter values for (a) $\nu=0.02$ and $D_{P}=0.0253$, (b) $\nu=0.02$ and $D_{P}=0.0053$, (c) $\nu=0.036$ and $D_{P}=0.0489$, (d) $\nu=0.036$ and $D_{P}=0.0089$, (e) $\nu=0.051$ and $D_{P}=0.3607$ and (f) $\nu=0.051$ and $D_{P}=0.3207$. See Table 2.1 for the other parameter values. $k_{0}=0$ and $k_{p}$ are wavenumbers corresponding to a local maximum of the dispersion relation. $k_{0}=0$ also corresponds to the plane wave perturbations.

We plot in Fig. 4 the dispersion relation (10) of the first coexistence equilibrium $P_{e, 1}, M_{e, 1}$ (cf. (4)), i.e. the lowest equilibrium branch in Figs. 1 and 2, for several combinations of parameter values. It is interesting to note the presence of non-zero $k$, denoted by $k_{p}$ in Fig. 4, that may yield $\lambda>0$ for $D_{P}$ above 
a critical value. The instability of the equilibrium for non-zero $k$ corresponds to a Turing instability, where a spatially periodic state will emerge.

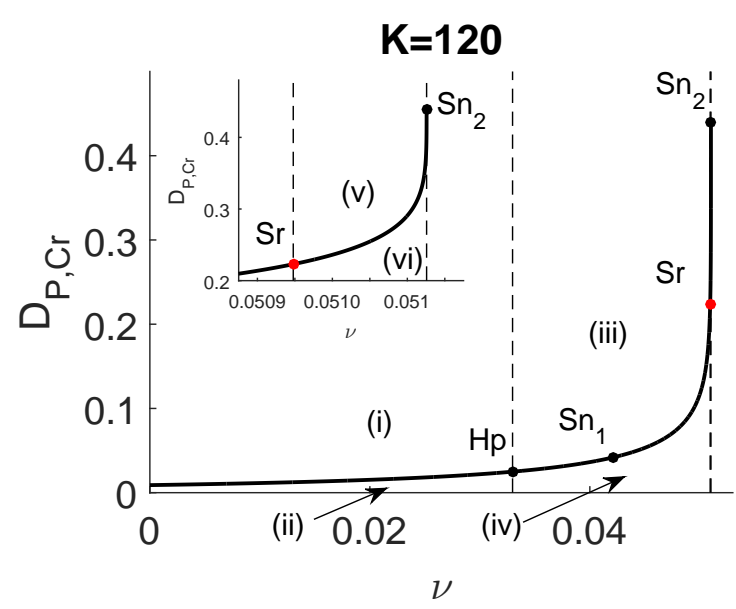

(a)

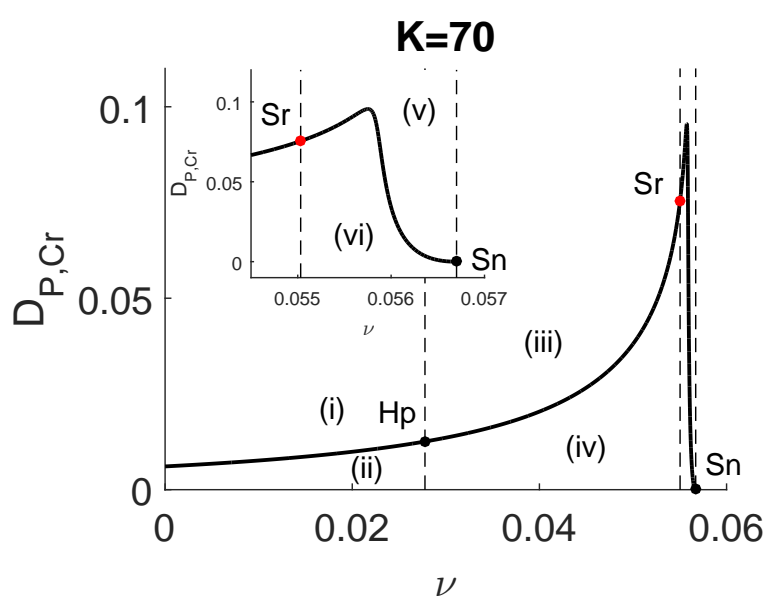

(b)

Fig. 5. The critical $D_{P}$ of the co-existence state $P_{e, 1}, M_{e, 1}$ (cf. (4)) for the appearance of unstable spectrum $\lambda$ with nonvanishing wavenumber $k$. See the text and Table 2 for the meaning of the dashed lines and the regions indicated by the Roman numerals.

Table 3. Description of the regions in Fig. 5 and their steady states.

\begin{tabular}{|c|c|c|c|c|}
\hline \multirow{2}{*}{ Region } & \multicolumn{3}{|c|}{ Uniform Solution } & \multirow{2}{*}{ Description } \\
\hline & $\operatorname{Re}(\lambda(0))$ & $\operatorname{Im}(\lambda(0))$ & $\operatorname{Re}\left(\lambda\left(k_{p}\right)\right)$ & \\
\hline (i) & $>0$ & $\neq 0$ & $<0$ & $\begin{array}{l}\text { Hopf bifurcation, stable } \\
\text { temporally periodic solutions }\end{array}$ \\
\hline (ii) & $>0$ & $\neq 0$ & $>0$ & $\begin{array}{l}\text { Hopf-Turing bifurcation, } \\
\text { spatio-temporally or spatially } \\
\text { periodic solutions }\end{array}$ \\
\hline (iii) & $<0$ & $\neq 0$ & $<0$ & stable uniform solutions \\
\hline (iv) & $<0$ & $\neq 0$ & $>0$ & $\begin{array}{l}\text { unstable uniform solution (Turing instability), } \\
\text { periodic states or } \\
\text { bloom in multistability interval }\end{array}$ \\
\hline (v) & $<0$ & $=0$ & $<0$ & stable uniform solutions \\
\hline (vi) & $<0$ & $=0$ & $>0$ & $\begin{array}{l}\text { unstable uniform solution (Turing instability), } \\
\text { bloom (equilibrium states) }\end{array}$ \\
\hline
\end{tabular}

Analysing the coexistence states in their existence region, we found that only $P_{e, 1}, M_{e, 1}$ can experience a Turing instability. Dispersion relations of the other equilibria have the shape of an inverted parabola, which does not allow the appearance of a local maximum at a non-zero $k$ and $D_{P}$ above a critical value.

By finding the largest eigenvalues (10) equal to zero and applying numerical continuation for varying $\nu$, we plot the critical value of the diffusion coefficient $D_{P, c r}$ below which one obtains bifurcations of spatially periodic states as shown in Fig. 5. The figure shows the stability region for $K=120$ and $K=70$. Our analysis yields six regions (i-vi) that are separated by the vertical dashed lines and the line of $D_{P, c r}$, in which one will obtain different qualitative characteristics. The behaviour of each region is summarised in Table 2 .

\subsection{Time evolution of the instability}

To illustrate the different dynamics behaviour in each region above, we will also present the time dynamics of the equilibrium perturbed in the unstable direction. The time integration of the governing equations 


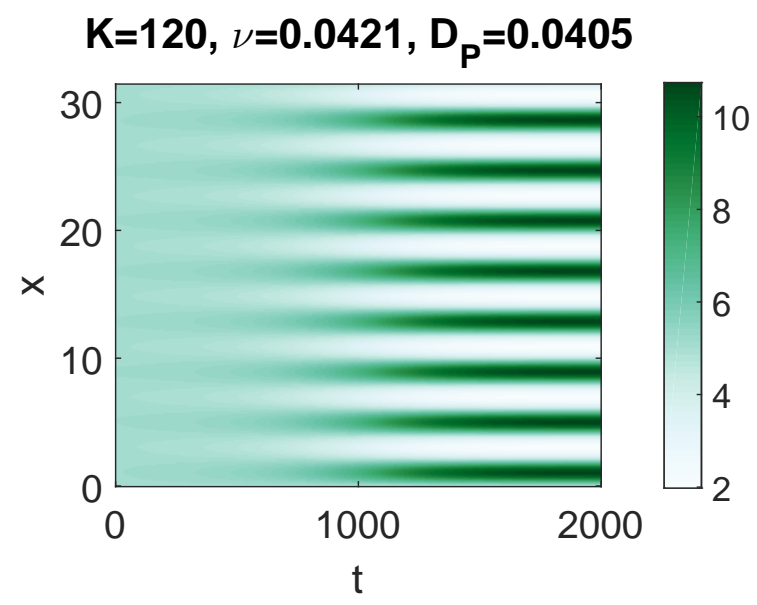

(a)

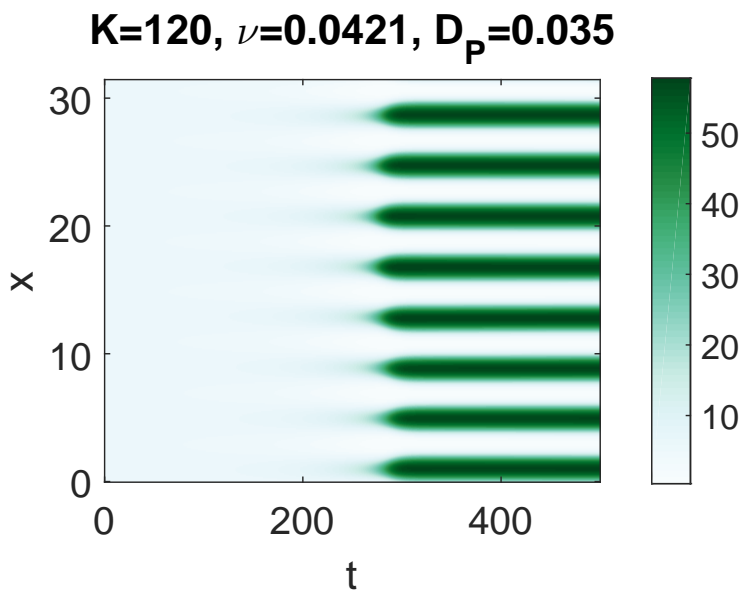

(c)

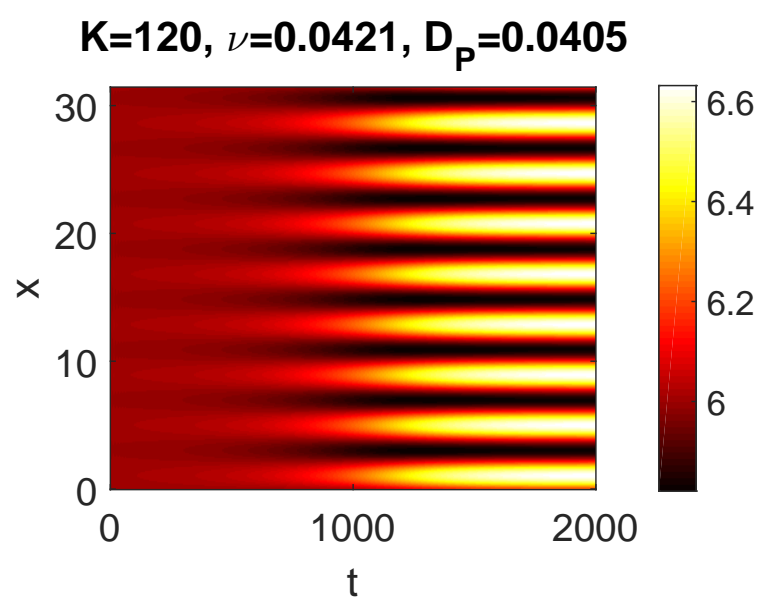

(b)

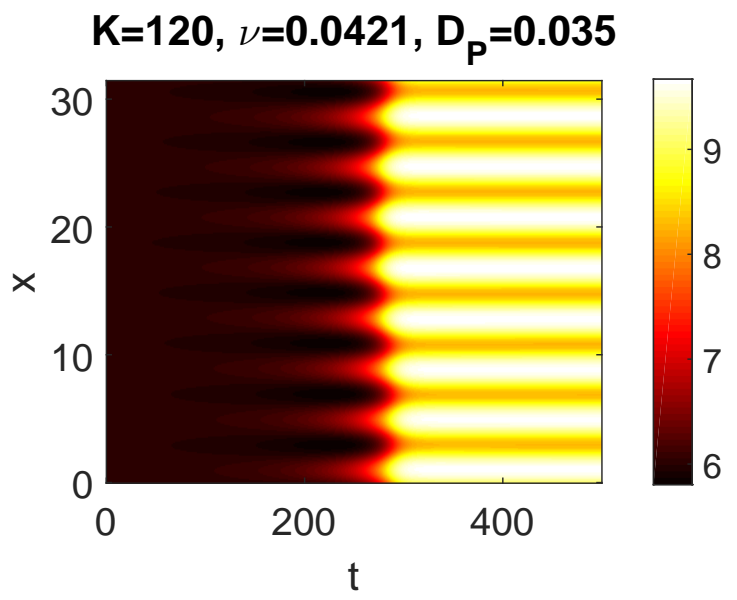

(d)

Fig. 6. Top view dynamics of a perturbed coexistence state for parameter values denoted in the title that correspond to region (iv) in Fig. 5(a), i.e., $D_{P}<D_{P, c r}$. and otherwise for panels (e-f). Left panels are for $P(x, t)$, while the right ones are for $M(x, t)$. Note that along the vertical and horizontal axes are the spatial $(x)$ and temporal $(t)$ variables, respectively.

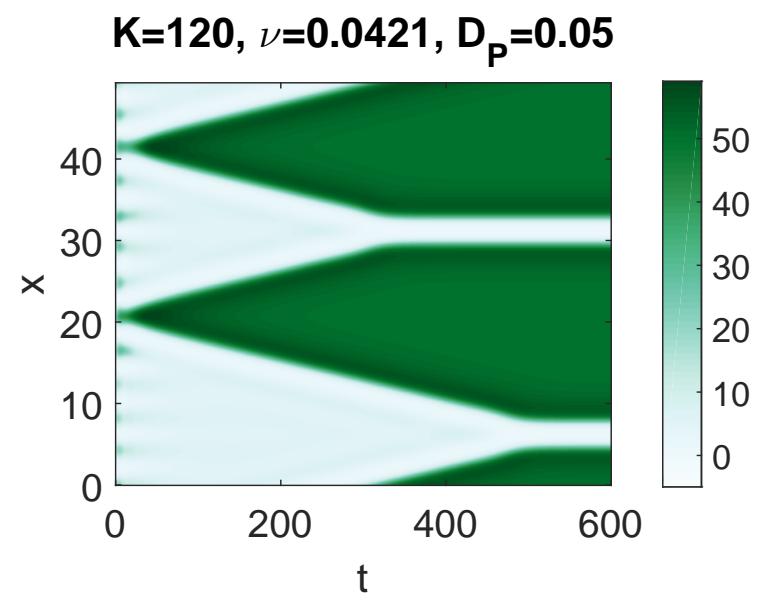

(a)

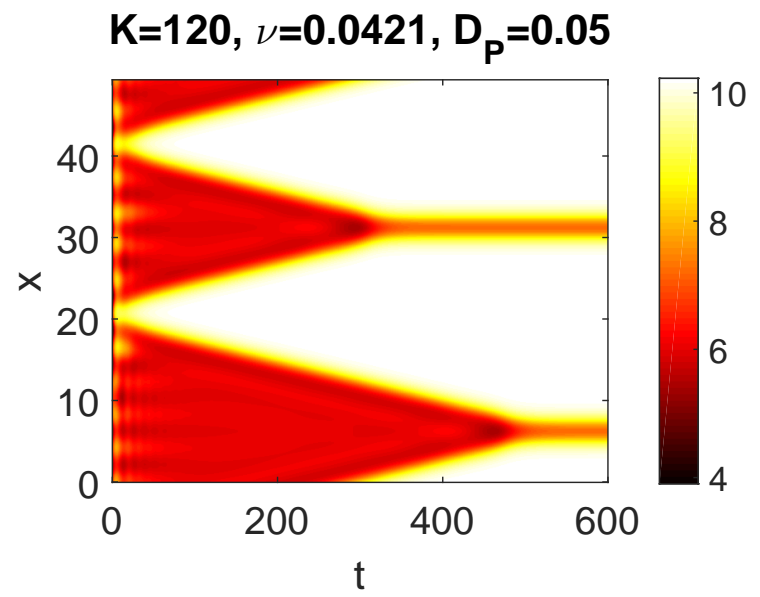

(b)

Fig. 7. Top view dynamics of a perturbed coexistence state for parameter values denoted in the title that correspond to region (iii) in Fig. 5(a), i.e., $D_{P}>D_{P, c r}$. Left panels are for $P(x, t)$, while the right ones are for $M(x, t)$. 


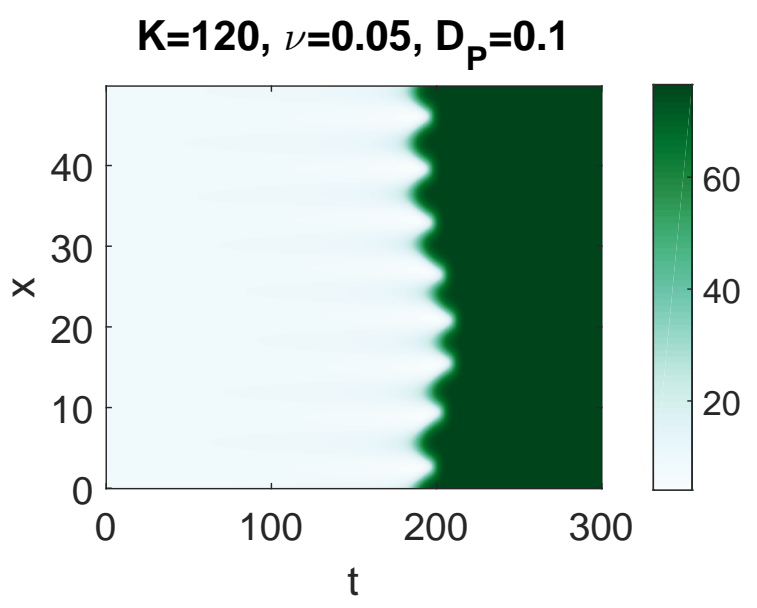

(a)

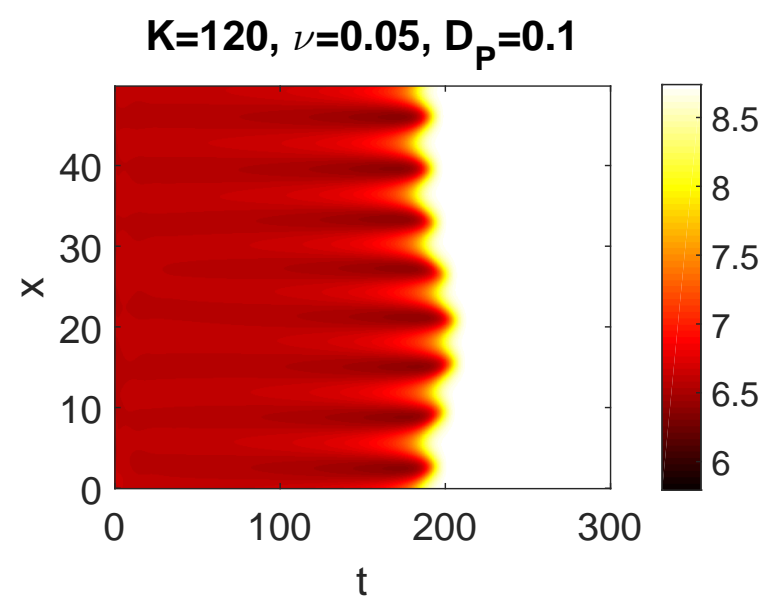

(b)

Fig. 8. The typical evolution of Turing instability in region (vi) close to the right saddle-node bifurcation $\left(S n_{2}\right)$. Left panels are for $P(x, t)$, while the right ones are for $M(x, t)$.

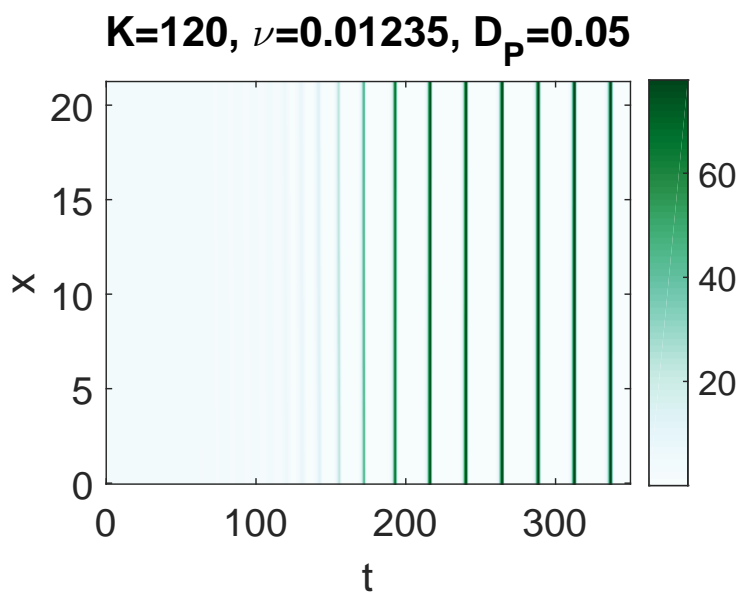

(a)

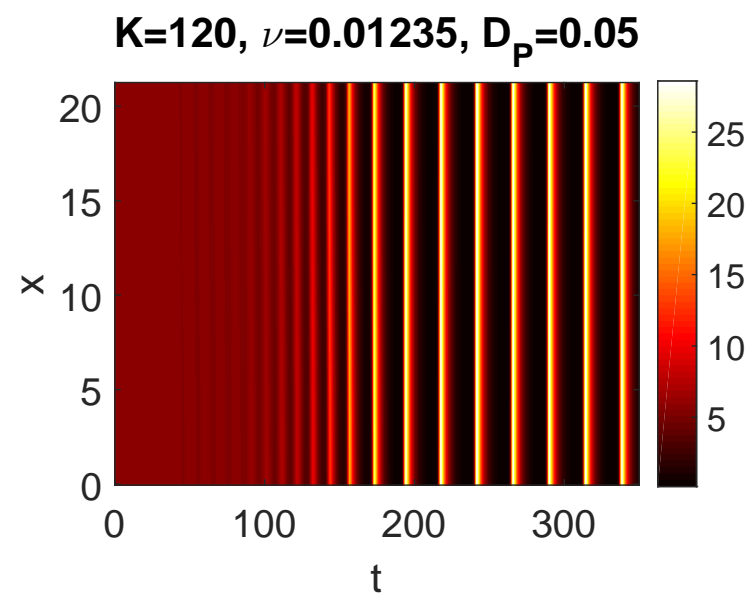

(b)

Fig. 9. Time dynamics of perturbed uniform solutions in region (i) of Fig. 5(a). Shown are the top view of the dynamics. Left panels are for $P(x, t)$, while the right ones are for $M(x, t)$.

(1) is performed using a Runge-Kutta method with the Laplacian being discretised using a central finite difference. We assume periodic boundary conditions because we are mainly interested in the bifurcations and dynamics of Turing patterns, which are spatially periodic solutions. One could also use Dirichlet or Neumann boundary conditions where provided that the computational domain is long enough, they should obtain similar results. The computational domain is taken to be a multiple of $2 \pi / k_{p}$, which is the characteristic wavelength of the bifurcating Turing patterns, i.e. $L=2 m \pi / k_{p}$ for $m \in \mathbb{Z}^{+}$. Typically we use $m=8$. Larger numbers have been checked as well where we did not notice any significant quantitative difference. As the initial condition, we use the uniform solutions that are perturbed randomly combined with perturbations in the direction of the most unstable eigenfunction. The parameter values are still as given in Table 2.1, with $r=1.5$ and $K, \nu$ and $D_{P}$ stated in the title of each panel.

Consider first, region (iv), in which the uniform solution is stable for $k=0$. At $k=k_{p}$, the solution can be unstable for $D_{P}$ less than the critical value. In Fig. 6 , we show the dynamics of the coexistence state that was stable in the ODE sense, but unstable in the presence of diffusion. One can note the creation of spatially periodic solutions for both $P$ and $M$. More interestingly, one should take a careful look at the 


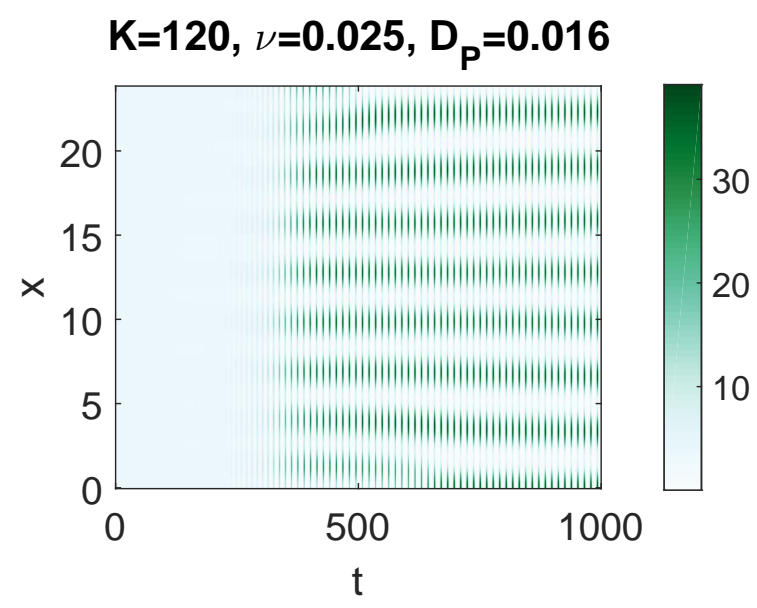

(a)

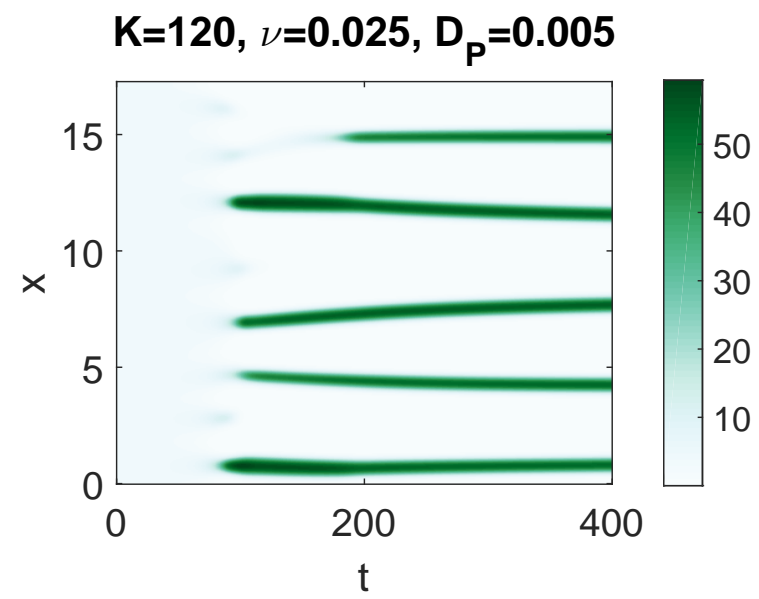

(c)

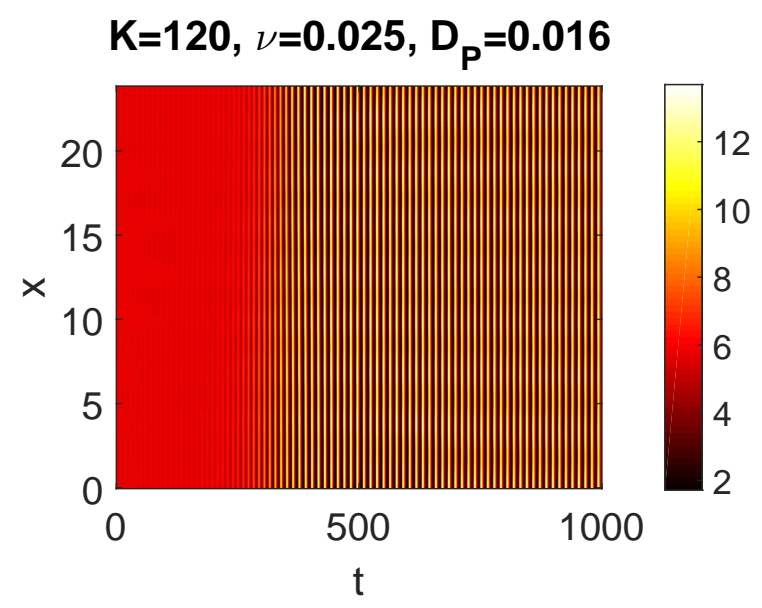

(b)

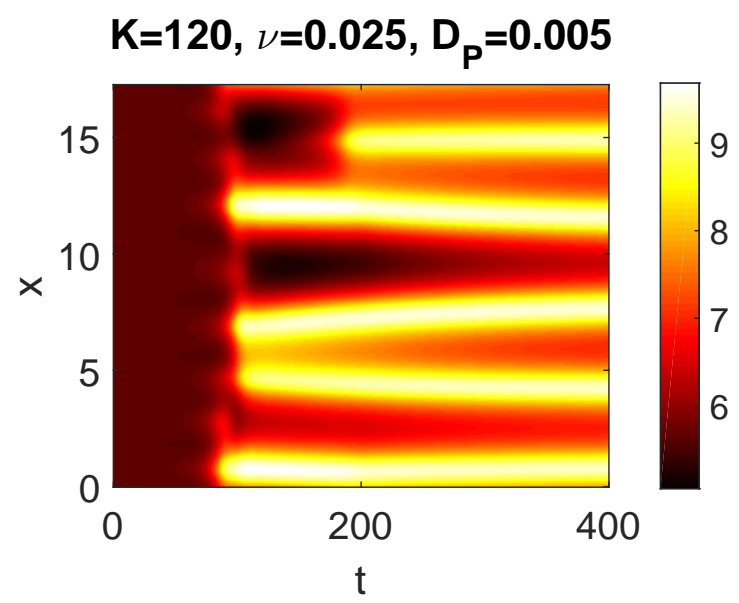

(d)

Fig. 10. Time dynamics of perturbed uniform solutions in region (ii) of Fig. 5(a) for different values of $D_{P}$. Shown are the top view of the dynamics. Left panels are for $P(x, t)$, while the right ones are for $M(x, t)$.

maximum of $P(x, t)$ when it reaches the steady state. Figs. 6(a)-6(d) that correspond to Turing instability show a big difference in the maxima that simply depend on $D_{P}$. While Figs. 6(a) and 6(b) show a rather small spatial oscillation around the equilibrium on the lower branch of Figs. 1(a) and 1(b), the steady states in Figs. 6(c) and 6(d) show the background plateau that is nothing else but the equilibrium on the upper branch in the multistability region in Figs. 1(a) and 1(b). Hence, we obtain a bloom formation with a spatial variation triggered by the presence of diffusion through Turing instability. We will show later that in this case there are indeed more than one kind of spatially periodic solutions, with one of them being unstable.

We also observe an interesting bloom formation that occurs in region (iii) that corresponds to stable equilibrium. Shown in Figs. 7(a) and 7(b) is the dynamics of the equilibrium with a rather large perturbation. In the beginning, one can see that the amplitude of the spatially periodic perturbation decreases (showing the stability of the background). Yet, at the same time some of the maxima keep increasing until they reach the value of the other stable equilibrium. The uniform solution then invades the remaining region. However, the process cannot continue and it stops at some time leaving bloom formations with spatial variations in the form of 'holes'. In the infinite domain, these 'holes' are likely to be solitary or localised solutions. They can also be viewed as 'coldspots' (i.e., phytoplankton and microzooplankton are scarce in localised areas). 
A different kind of bloom formation in the model with diffusion is exhibited by the dynamics in region (vi). While region (v) only yields stable equilibrium, we show in Fig. 8, the typical evolution of the unstable uniform due to Turing instability. Unlike Fig. 6, here we obtain a bloom formation without any spatial variation. The reason is not immediately clear from the time dynamics. In this case, one needs a bifurcation diagram of the steady state, which will be discussed further in the following subsection. We will know later that for the particular parameter values taken in the figure, the governing equations (1) do not admit spatially periodic solutions.

A more interesting case is perhaps the regions (i,ii), in which we have unstable uniform solutions with the Hopf instability corresponding to the perturbation with $k=0$. By performing a numerical integration for $D_{P}$ above the critical value, i.e. region (i), indeed as time evolves the uniform solution becomes oscillating, see Figs. 9(a) and 9(b). The maximum and minimum amplitude of the oscillation do coincide with those given in Fig. 1(a) and 1(b).

In region (ii), not only the instability due to Hopf, we also have an instability due to Turing, i.e. Turing-Hopf instability. As shown in Figs. 10(a) and 10(b), we indeed obtain patterns that are periodic both temporally as well spatially. Interestingly if we take a much smaller value of $D_{P}$ we have a timeindependent solution. This is shown in Fig. 10(c), 10(d). We can view the steady state as a collection of 'hotspots', where phytoplankton and microzooplankton are abundant in localised areas.

Performing the same simulations for $K=70$ (not presented here), we do not obtain any bloom formation over the range of $\nu$ considered (see Fig. 5(b)), and there is no hysteresis in the system in this case.

\subsection{Bifurcation diagram of time-independent patterns}

After depicting several qualitatively different dynamics within the different regions, it is then instructive to consider the existence and stability of time-independent spatially periodic solutions. This will explain and provide unified pictures of the observed patterns and their development reported in Subsection 4.2 above.

To obtain bifurcation diagrams of the solutions, we solve the time-independent governing equations (1) numerically using a Newton method. We use steady states of our time dynamic simulations as an initial guess of the iteration method. Again, the Laplacian terms are discretised using a finite difference scheme with periodic boundary conditions. When a time-independent solution, let's say $P_{s}$ and $M_{s}$, is obtained, we also determine numerically its linear stability by solving the corresponding linear eigenvalue problem:

$$
\begin{aligned}
& \lambda \tilde{P}=D_{P} \frac{\partial^{2} \tilde{P}}{\partial x^{2}}+\left(r-\frac{2 r P_{s}}{K}-\frac{a M_{s}-\frac{a b P_{s} M_{s}}{1+b P_{s}}}{1+b P_{s}}\right) \tilde{P}-\frac{a P_{s}}{1+b P_{s}} \tilde{M} \\
& \lambda \tilde{M}=D_{M} \frac{\partial^{2} \tilde{M}}{\partial x^{2}}+\left(\frac{a M_{s}-\frac{a b P_{s} M_{s}}{1+b P_{s}}}{1+b P_{s}}\right)\left(\gamma-\nu M_{s}\right) \tilde{P}+\frac{a \gamma-2 \nu M_{s}}{1+b P_{s}} P_{s} \tilde{M}-m \tilde{M}
\end{aligned}
$$

that is obtained from substituting the perturbation ansatz $P=P_{s}(x)+\tilde{P}(x) e^{\lambda t}$ and $M=M_{s}(x)+\tilde{M}(x) e^{\lambda t}$ into the governing equations (1) and linearising about $\|\tilde{P}\|,\|\tilde{M}\| \ll 1$. Again, the solution, i.e., $P_{s}$ and $M_{s}$, is called unstable if and only if there is an eigenvalue with $\operatorname{Re}(\lambda)>0$ and linearly stable otherwise.

In this section, $D_{P}$ will be our control parameter. As it is varied, there can be a saddle-node bifurcation. To continue the computations past such a turning point, we use a pseudo-arclength method [Keller, 1987; Seydel , 2009]. When, upon a continuation, we obtain a branch with unstable corresponding solutions, we then evolve them using our time-integration code to (expectedly) obtain another spatially periodic steady state. Computing the new (stable) solution using the continuation code will yield another bifurcation curve that possibly is related to the previously computed solution branches. Performing these steps repeatedly will yield a rather complete bifurcation diagram of time-independent patterns of the system.

Bifurcation diagrams from the steady states of the dynamics reported in Section 4.2 are summarised in Figs. 11 and 12. For simplicity, herein we only plot the variable $P$. Note that from the critical point $D_{P, c r}$, there is a bifurcation of periodic states, i.e., Turing bifurcations, that can be either supercritical or subcritical. 


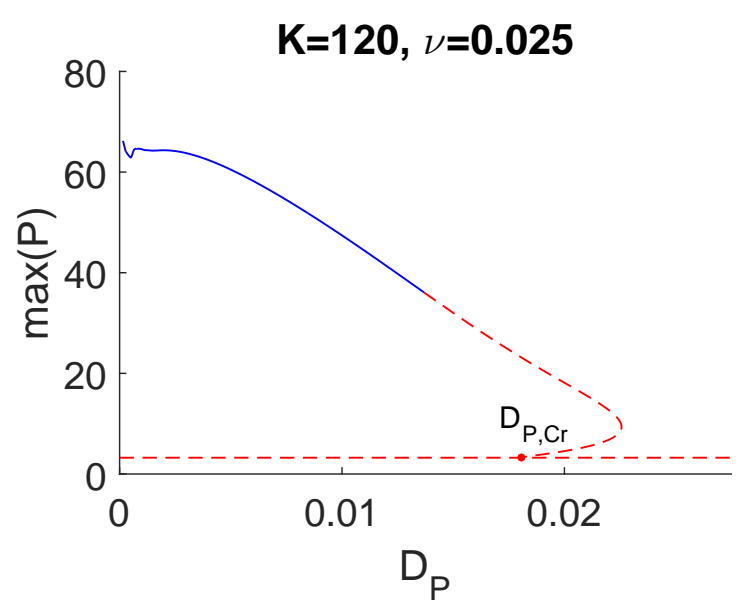

(a)

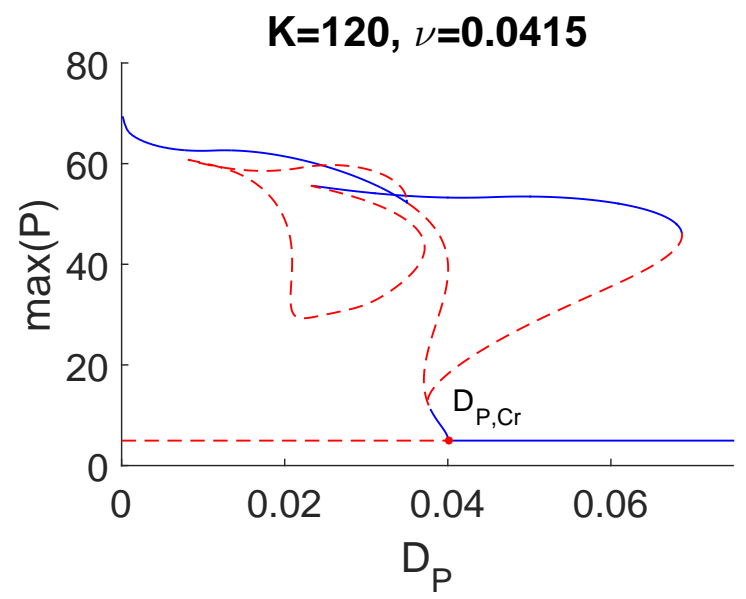

(c)

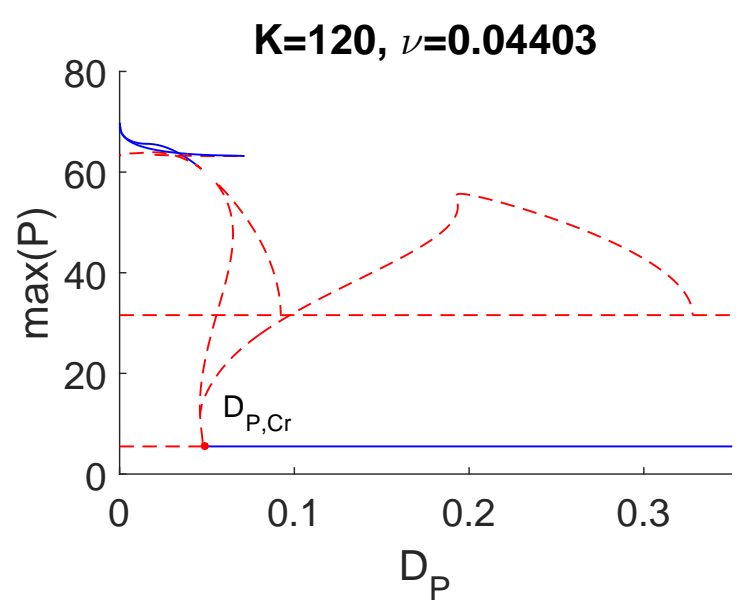

(e)

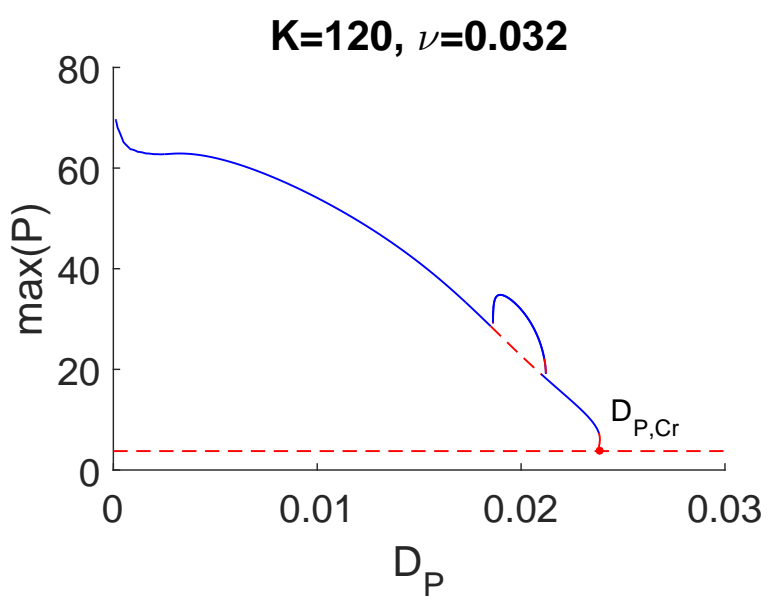

(b)

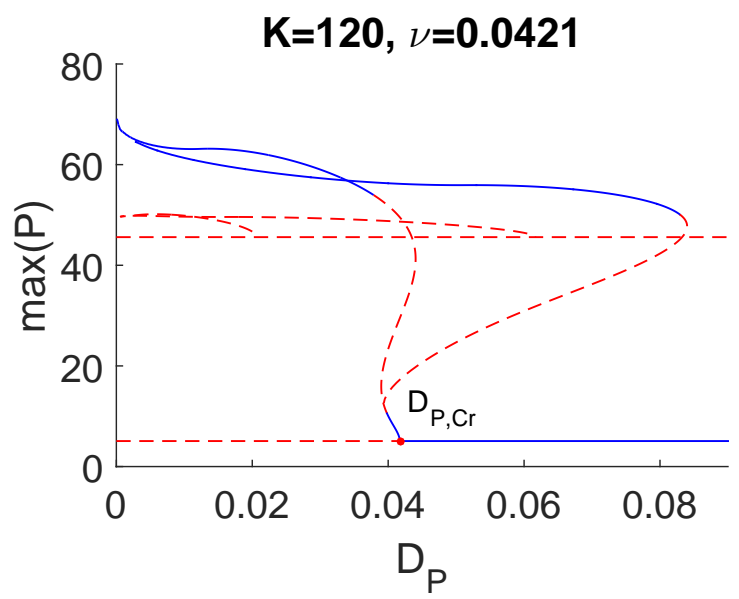

(d)

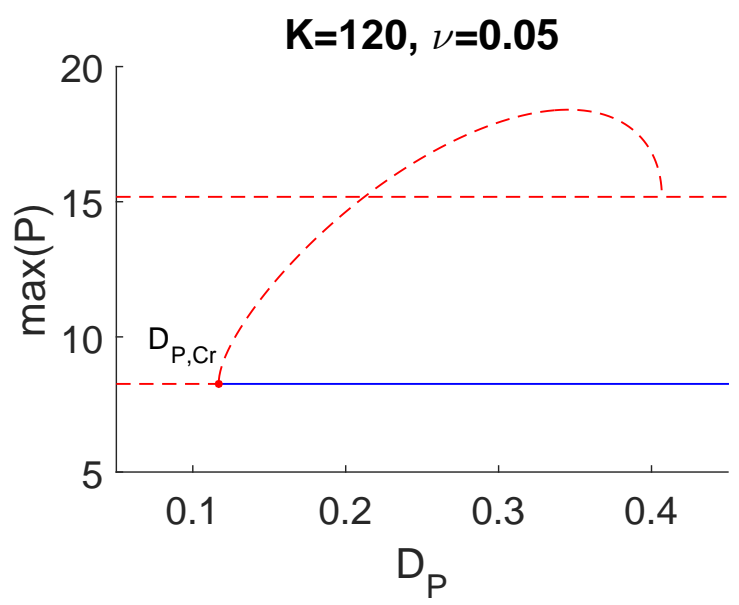

(f)

Fig. 11. Bifurcation diagrams of periodic solutions that emanates from Turing instability with $K=120$ for several parameter values of $\nu$. Red dashed line shows instability. The additional horizontal line in Figs. 11(d)-11(f) is the unstable equilibrium solution existing between $S n_{1}$ and $S n_{2}$ in Fig. 1(a) that is connected in the bifurcation diagrams to $P_{e, 1}$ by spatially periodic states. 
First, let us start with region (ii) of Fig. 5(a). The typical time dynamics of solutions within the regions are represented by Fig. 10. Using the steady state in Figs. 10(c) and 10(d) as an initial guess for our Newton algorithm, we obtain a time independent spatially periodic state of the system for the same parameter values. We then vary $D_{P}$ from which we obtain the bifurcation diagram in Fig. 11(a) for $\nu$ below the Hopf point. We obtain that Figs. 10(c) and 10(d) correspond to the value of $D_{P}$ with stable spatially periodic states. Using the bifurcation diagram, we can also explain the dynamics in Fig. 10(a) and 10 (b) that for the same parameter values as in the figures, the governing equations do have a time independent, but unstable, spatially periodic solution. The instability apparently leads to a limit cycle of spatially periodic states, i.e., a Hopf-Turing pattern.

The bifurcation diagram in Fig. 11(a) tells us that the spatially uniform states $P_{e, 1}$ and $M_{e, 1}$ loose their stability to spatially periodic solutions via a subcritical pitchfork bifurcation. This implies that even in region (i) of Fig. 5(a), we can have spatially periodic solutions, but they are unstable. This conclusion applies to the dynamics in Fig. 9 as well, even though it is for a slightly different value of $\nu$.

We show in Figs. 11(b) and 11(c) the change in the bifurcation diagrams as the parameter $\nu$ increases towards the Hopf point $H_{P}$. Two important features that need to be noted are that the Turing (pitchfork) bifurcation of periodic solutions change from subcritical to supercritical and there are intervals of $D_{P}$ where one can obtain coexistence of more than one stable periodic solutions. The multi stability is related to the bistability region of the uniform solutions shown in Figs. 1 and 3. The diagram becomes complicated as $\nu$ increases further.

Bifurcation diagrams of the steady states from the time dynamics in Figure 6 are presented in Fig. 11(d). The creation of coldspots for $D_{P}$ above the Turing point $D_{P, C r}$ in Fig. 7 can also be explained using the bifurcation diagram in Fig. 11(d). Such a pattern formation occurring not in the Turing regime $D_{P}<D_{P, C r}$ is because the spatially periodic solutions also extend to the region $D_{P}>D_{P, C r}$. However, to achieve such an interesting steady state one requires large perturbations as $P_{e, 1}$ and $M_{e, 1}$ are stable in that region (hence it is beyond the linear stability analysis).

Finally, bifurcation diagrams of spatially periodic states in region (vi) of Fig. 5(a), that is represented by time dynamics in Fig. 8, are presented in Fig. 11(f). It is interesting to note that the Turing bifurcation is subcritical again here. Moreover, there is no stable periodic state, which implies that the Turing instabilities will force the system towards the stable uniformly equilibrium state, i.e. bloom formations without any spatial variation as obtained in Fig. 8 .

We have also computed bifurcation diagrams of spatially periodic solutions with $K=70$ that represent some of the regions in Fig. 5(b). Figure 12 presents them for several values of $\nu$. In general, they are similar to those with $K=120$. The main difference is that for $K=70$, all the bifurcations of the periodic states are supercritical and hence no hysteresis is observed, which is in agreement with our time-dynamics simulations (not presented here).

\section{Conclusion}

We have studied the infochemical mediated predator-prey model first introduced by [Lewis et al., 2012] to explore the interactions and dynamics of a simple microzooplankton and phytoplankton foodweb with external copepod predation. We have provided a mathematical explanation for the numerical results first reported by [Lewis et al., 2012], and have further extended their parameter study to also consider the role of the phytoplankton carrying capacity, $K$. We have shown how the general system stability is dependent on $K$ and $\nu$ and explored over what range phytoplankton formation can occur.

We have also extended the model of [Lewis et al., 2012] to explore spatial diffusion in the horizontal plane. We have highlighted the regions of parameter space where a Turing instability can occur and illustrated how periodic spatial patterns develop within the system. We have also demonstrated that timedependent spatial patterns can become unstable temporally.

While many, if not all, of previous works consider vertical motility (see, e.g., [Huisman \&et al., 2006; Zagaris et al., 2009; Zagaris \& Doelman, 2011; Sewalt et al., 2015]), our paper proposes horizontal movement. One particularly different result we presented here is the observation of localised solutions that biologically may indicate the presence of hotspots (such as those in Figs. 10(c) and 10(d)) or coldspots 


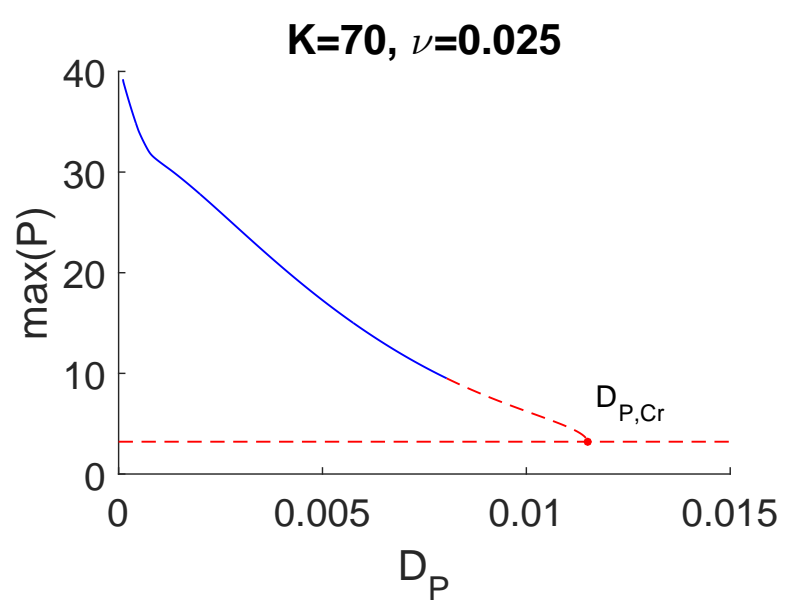

(a)

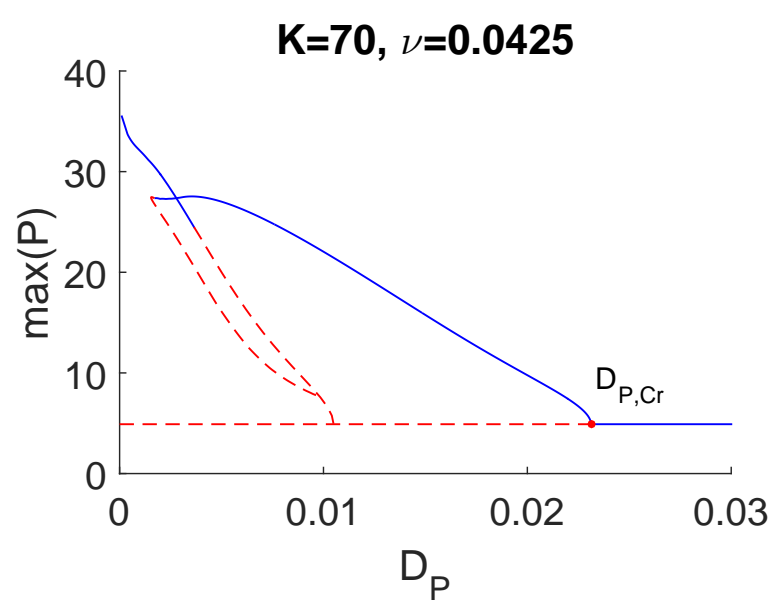

(c)

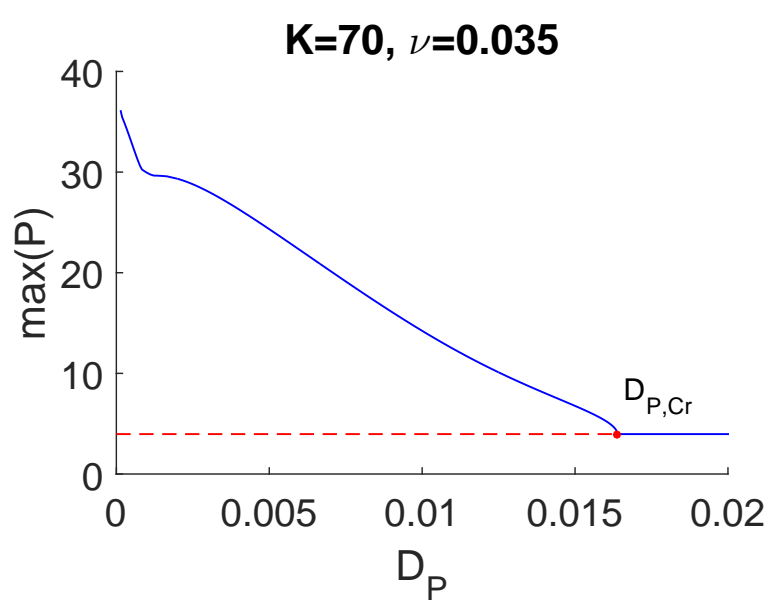

(b)

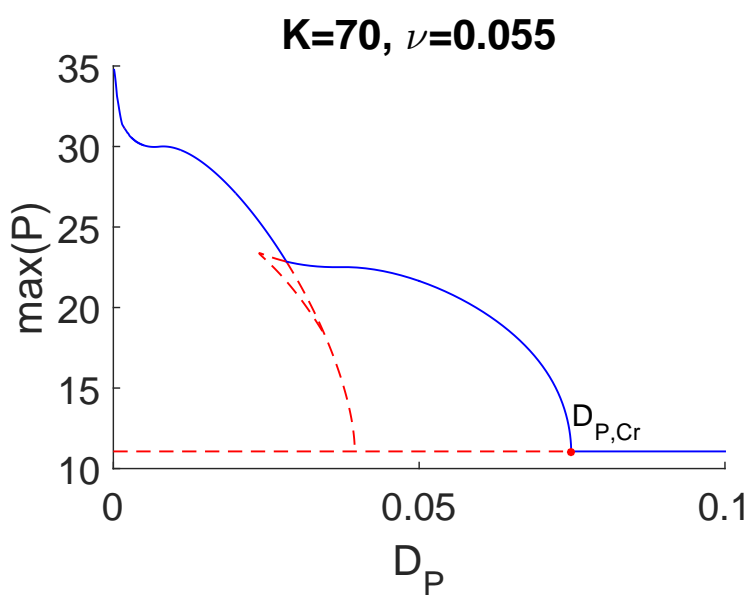

(d)

Fig. 12. Bifurcation diagrams of periodic solutions that emanates from Turing instability with $K=70$ for several parameter values of $\nu$.

(such as those shown in Figs. 7(a) and 7(b)). Our study can considerably serve as a blueprint for the analytic investigation of plankton dynamics with horizontal mobility. It will be interesting to study the existence and stability of the spot solutions using geometric techniques as employed in, e.g., [Harley et al., 2014, 2015]. Studying whether such solutions persist in two-dimension and become actual coldspots or hotspots (which are localised in both directions) or 'cold-stripes' and 'hot-stripes' (which are localised in one direction but constant in the perpendicular one), see, e.g., [Banerjee \& Volpert, 2017; Banerjee et al., 2018; Rodrigues et al., 2011], is also intriguing and addressed for future work. Additionally, our analysis in this paper is concentrated on standing patterns. It will be particularly interesting to extend the work to consider the possibility of travelling waves as well as spatiotemporal chaos.

It is important to note that the spatiality in (1) was introduced by directly adding diffusion terms to the simple model proposed in [Lewis et al., 2012]. At this stage, we have not analysed the order of the diffusion coefficients compared to the other parameter values to be biologically and physically relevant. Our model is also constructed by assuming a stable copepod population. These assumptions and limitations are addressed for future work, which include, e.g., a more realistic model extension.

\section{Acknowledgement}

We thank the two anonymous referees for their very careful reading that improved the manuscript. 


\section{References}

Jennings S, Kaiser M and Reynolds JD (2009) Marine fisheries ecology, John wiley \& Sons.

Sherr E, Sherr B,(1988) Role of microbes in pelagic food webs: a revised concept, Limnology and oceanography, vol.33:1225-1227, No. 5, Wiley Online Library.

Klein Breteler WCM, Schogt N, Baas M, Schouten S, Kraay GW (1999) Trophic upgrading of food quality by protozoans enhancing copepod growth: role of essential lipids. Mar biol 135:191198.

Hansen FC, Reckermann M, Klein Breteler WCM, Riegman R (1993) Phaeocystis blooming enhanced by copepod predation on protozoa: evidence from incubation experiments. Mar ecol prog ser 102:5157.

Vos M, Vet LEM, Wackers FL, Middelburg JJ, van der Putten WH, Mooij WM, Heip CHR, van Donk E (2006) Infochemicals structure marine, terrestrial and freshwater food webs: implications for ecological informatics. Ecol inform 1:2332.

Pohnert G, Steinke M, Tollrian R (2007) Chemical cues, defence metabolites and the shaping of pelagic interspecific interactions. Trends ecol evol 22:198204.

Hay ME,(2009) Marine chemical ecology: chemical signals and cues structure marine populations, communities, and ecosystems. Annu Rev Mar Sci 1:193212.

Nevitt GA, Veit RR, Kareiva P (1995) Dimethyl sulphide as a foraging cue for Antarctic Procellariiform seabirds. Nature 376:680682.

Kowalewsky S, Dambach M, Mauck B, Dehnhardt G (2006) High olfactory sensitivity for dimethyl sulphide in harbour seals. Biol Lett 2:106109.

Steinke M, Stefels J, Stamhuis E (2006) Dimethyl sulphide triggers search behaviour in copepods. Limnol oceanogr 51:19251930.

Wolfe, Gordon V, (2000) The chemical defense ecology of marine unicellular plankton: constraints, mechanisms, and impacts, The Biological bulletin, vol 198:225-244, No. 2, Marine biological laboratory.

Steinke, M, Malin G, Liss PS, (2002) Trophic interactions in the sea: an ecological role for climate relevant volatiles, Journal of phycology, vol. 38:630-638,, No. 4, Wiley Online Library.

Charlson RJ, Lovelock JE, Andreae MO, Warren SG, (1987) Oceanic phytoplankton, atmospheric sulphur, cloud albedo and climate. Nature 326:655661.

Lewis ND, Breckels MN, Archer SD, Morozov A, Pitchford JW, Steinke M, Codling, EA, (2012) Grazinginduced production of DMS can stabilize food-web dynamics and promote the formation of phytoplankton blooms in a multitrophic plankton model. Biogeochemistry 110, 303-313.

Rosenzweig ML, MacArthur RH (1963) Graphical representation and stability conditions of predatorprey interactions. Am Nat 97:209223.

Lewis ND, Breckels MN and Steinke M and Codling EA, (2013) Role of infochemical mediated zooplankton grazing in a phytoplankton competition model, Ecological complexity, vol 16:41-50, Elsevier.

Heuschele J, Selander E, (2014) The chemical ecology of copepods. Journal of plankton research, vol 36: 895-213.

Calbet A, (2001) Mesozooplankton grazing effect on primary production: a global comparative analysis in marine ecosystems. Limnol oceanogr 46:18241830.

Kot M, (2001) Elements of mathematical ecology, Cambridge University Press.

Huisman J, Thi N N P, David M K and Sommeijer B, (2006) Reduced mixing generates oscillations and chaos in the oceanic deep chlorophyll maximum, Nature 439, 322325, Nature publishing group.

Zagaris A, Doelman A, Thi N N P and Sommeijer, B P, (2009) Blooming in a non-local, coupled phytoplanktonnutrient model, SIAM J. Appl. Math. 69, 11741204, SIAM.

Zagaris A and Doelman A, (2011) Emergence of steady and oscillatory localized structures in a phytoplankton-nutrient model, Nonlinearity 24, 3437-3486.

Sewalt L, Doelman A, Meijer H G E, Rottschäfer V and Zagaris A, (2015) Tracking pattern evolution through extended center manifold reduction and singular perturbations, Phys. D 298-299, 48-67, Elsevier.

Malchow H, Petrovskii SV and Venturino E, (2008) Spatiotemporal patterns in ecology and epidemiology: theory, models, and simulation, Chapman \& Hall/CRC Press London.

Feudel U, (2008) Pattern Formation in Marine Systems, Complexity and Synergetics, vol. 3:179-196, 
Springer.

Ghorai S and Poria S, (2016) Pattern formation and control of spatiotemporal chaos in a reaction diffusion prey-predator system supplying additional food, Chaos, Solitons \& Fractals, vol 85:57-67, Elsevier.

Roelke DL, Eldridge PM and Cifuentes LA, (1999) A model of phytoplankton competition for limiting and nonlimiting nutrients: implications for development of estuarine and nearshore management schemes, Estuaries and Coasts, vol 22:92-104, Springer.

Hammer, Astrid C and Pitchford, Jonathan W, (2005) The role of mixotrophy in plankton bloom dynamics, and the consequences for productivity, vol 62:833-840, No. 5, ICES Journal of Marine Science, Oxford University Press.

Pitchford J and Brindley J, (1998) Intratrophic predation in simple predator-prey models, Bulletin of mathematical biology, vol 60: 937-953, Springer.

Nickalls R, (1993) A new approach to solving the cubic: Cardan's solution revealed. The Mathematical Gazette, 354-359.

Jankovic, M and Petrovskii S, (2014) Are time delays always destabilizing? Revisiting the role of time delays and the Allee effect. Theoretical Ecology 7(4):335-349.

Sieber M, Hilker FM, (2012) The hydra effect in predator-prey models. J Math Biol. 64:341-60, Springer.

Harvey EL and Menden-Deuer S, (2012) Predator-induced feeding behaviors in phytoplankton: a new mechanism for harmful algal bloom formation? PLoS One 7, e46438, Public Library of Science.

Kiørboe T, Jiang H, Gonçalves RJ, Nielsen LT, Wadhwa N, (2014) Flow disturbances generated by feeding and swimming zooplankton, Proceedings of the National Academy of Sciences 111, 1173811743, National Acad Sciences.

Edwards and Brindley, John, Andrew M, (1999) Zooplankton mortality and the dynamical behaviour of plankton population models. Bulletin of mathematical biology, 61:303-339, Springer.

Franks, Peter JS, (2002) NPZ models of plankton dynamics: their construction, coupling to physics, and application. Journal of Oceanography, 58:379-387, Springer.

Morozov, Andrew and Arashkevich, Elena and Nikishina, Anastasia and Solovyev, Konstantin, (2010) Nutrient-rich plankton communities stabilized via predator-prey interactions: revisiting the role of vertical heterogeneity, Mathematical medicine and biology: Journal of the IMA, 28:185-215, Oxford University Press.

Saiz, Enric and Calbet, Albert, (2007) Scaling of feeding in marine calanoid copepods. Limnology and Oceanography, 52:668-675, Wiley Online Library.

Hansen B. and Tande, KS and Berggreen, UC, (1990) On the trophic fate of Phaeocystis pouchetii (Harlot). III. Functional responses in grazing demonstrated on juvenile stages of Calanus finmarchicus (Copepoda) fed diatoms and Phaeocystis. Journal of Plankton Research, 12:1173-1187, Oxford Univ Press.

Edwards, Andrew M and Yool, Andrew, (2000) The role of higher predation in plankton population models. Journal of Plankton Research, 22:1085-1112, Oxford Univ Press.

Keller HB, (1987) Lectures on numerical methods in bifurcation problems, Notes by AK. Nandakumaran \& M. Ramaswamy, Indian Institute of Science, Bangalore, Springer-Verlag, Berlin.

Seydel, R. (2009). Practical bifurcation and stability analysis (Vol. 5). Springer science \& business media. Chicago

Harley K, van Heijster P and Pettet GJ, (2014) A geometric construction of travelling wave solutions to the Keller-Segel model, Anziam J. 55, 399415.

Harley K, van Heijster P, Marangell R, Pettet GJ, and Wechselberger M, (2015) Numerical computation of an Evans function for travelling waves, Math. Biosci. 266, 3651.

Banerjee M, \& Volpert V. (2017). Spatio-temporal pattern formation in RosenzweigMacarthur model: effect of nonlocal interactions. Ecological complexity, 30, 2-10.

Banerjee, M., Ghorai, S., \& Mukherjee, N. (2018). Study of cross-diffusion induced Turing patterns in a ratio-dependent prey-predator model via amplitude equations. Applied mathematical modelling, 55, 383-399.

Rodrigues, L. A. D., Mistro, D. C., \& Petrovskii, S. (2011). Pattern formation, long-term transients, and the TuringHopf bifurcation in a space-and time-discrete predatorprey system. Bulletin of mathematical 
20 REFERENCES

biology, 73(8), 1812-1840. 